\title{
Metformin attenuates the D-galactose-induced aging process via the UPR through the AMPK/ERK1/2 signaling pathways
}

\author{
HUA CAI $^{1 *}$, BAOAI HAN $^{1 *}$, YUJUAN HU $^{1 *}$, XUEYAN ZHAO $^{1}$, ZUHONG HE $^{1}$, XUBO CHEN $^{1}$, HAIYING SUN $^{1}$, \\ JIE YUAN $^{1}$, YONGQIN LI ${ }^{1}$, XIUPING YANG ${ }^{1}$, WEN KONG $^{2}$ and WEI-JIA KONG ${ }^{1}$
}

Departments of ${ }^{1}$ Otorhinolaryngology and ${ }^{2}$ Endocrinology, Union Hospital, Tongji Medical College, Huazhong University of Science and Technology, Wuhan, Hubei 430022, P.R. China

Received May 7, 2019; Accepted September 18, 2019

DOI: $10.3892 / \mathrm{ijmm} .2020 .4453$

\begin{abstract}
Age-related hearing loss, also termed central presbycusis, is a progressive neurodegenerative disease; it is a devastating disorder that severely affects the quality of life of elderly individuals. Substantial evidence has indicated that oxidative stress and associated protein folding dysfunction have a marked influence on neurodegenerative diseases. In this study, we aimed to cells to investigate whether metformin protects against age-related pathologies and to elucidate the underlying mechanisms; specifically, we focused on the role of unfolded protein response (UPR) via the AMPK/ERK1/2 signaling pathways. For this purpose, the biguanide compound,
\end{abstract}

Correspondence to: Professor Wei-Jia Kong, Department of Otorhinolaryngology, Union Hospital, Tongji Medical College, Huazhong University of Science and Technology, 1277 Jiefang Avenue, Wuhan, Hubei 430022, P.R. China

E-mail: entwjkong@hust.edu.cn

Dr Wen Kong, Department of Endocrinology, Union Hospital, Tongji Medical College, Huazhong University of Science and Technology, 1277 Jiefang Avenue, Wuhan, Hubei 430022, P.R. China

E-mail: wenly-kong@163.com

*Contributed equally

Abbreviations: D-gal, D-galactose; ABR, auditory brainstem response; mtDNA, mitochondrial DNA; ROS, reactive oxygen species; CD, common deletion; SOD, superoxide dismutase; GSH, glutathione; MDA, malondialdehyde; TEM, transmission electron microscopy; qPCR, quantitative polymerase chain reaction; MT, mitochondrial; ER, endoplasmic reticulum; UPR, unfolded protein response; Foxo, forkhead transcription factor, class O transcription; AMPK, AMP-activated protein kinase; ERK, extracellular signal-regulated kinase; HSP90, heat shock protein 90; HSP60, heat shock protein 60; CHOP, C/EBP homologous protein; GRP78, glucose-regulated protein 78; TUNEL, terminal deoxynucleotidyl transferase-mediated deoxyuridine 5'-triphosphate nick-end labeling; ANOVA, analysis of variance

Key words: aging, metformin, auditory cortex, UPR, AMPK, ERK1/2 metformin, a medication widely used in the treatment of type 2 diabetes, was administered to rats in a model of mimetic aging. In addition, senescent PC12 were treated with metformin. Although it has been well established that UPR signaling is activated in response to cellular stress and is associated with the pathogenesis of neuronal deterioration, the detailed functions of the UPR in the auditory cortex remain unclear. We found that metformin treatment markedly affected the UPR and the AMPK/ERK1/2 signaling pathway, and maintained the auditory brainstem response (ABR) threshold during the aging process. The results indicated that the regulation of the UPR and AMPK/ERK1/2 signaling pathway by metformin significantly attenuated hearing loss, cell apoptosis and age-related neurodegeneration. Reversing these harmful effects through the use of metformin suggests its involvement in restoring the antioxidant status and protein homeostasis related to the underlying pathology of presbycusis. The findings of this study may provide a better approach for the treatment of age-related neurodegeneration diseases.

\section{Introduction}

Aging is a normal physiological process that not only increases susceptibility to diseases, but also gradually reduces physiological functions (1). Age-related hearing loss (AHL), also referred to as presbycusis, is a type of sensorineural deafness with bilateral high-frequency hearing loss that progressively declines with age (2). Aging-related changes in the central auditory system have a negative impact on auditory perception and verbal communication, or both (3). However, the mechanisms involved in central presbycusis remain unclear.

A number of aging pathways are closely related to cellular protein homeostasis, suggesting that maintaining a healthy protein homeostasis is crucial for longevity (4). Accumulating lines of evidence have indicated that the unfolded protein response (UPR) is the link between aging and proteostasis in a variety of vital organisms $(4,5)$. Hearing loss caused by both environmental and genetic factors is improved by regulating the UPR (6). The continuous aggravation of the endoplasmic reticulum (ER) and mitochondrial (MT) stress participates in the pathogenesis of AHL. Heat shock protein (HSP)- or stress-related chaperones have been identified as markers of the UPR, and a number of studies have been conducted 
mainly on the UPR signaling pathways (7-9). However, the specific mechanisms and proteostatic UPR signaling pathways involved in age-related diseases remain unknown.

Metformin, an AMP-activated protein kinase (AMPK) activator, is a medication used in the first-line treatment of type 2 diabetes. Metformin readily crosses the blood-brain barrier and is distributed to various regions in the brain (10), and recently, it has been reported to promote neurogenesis, thereby providing potential neuroprotection (11). However, whether metformin interacts with the UPR and the exact role of metformin in the UPR remain unclear. It is well known that AMPK/ERK1/2 are growth factor signals and important metabolic regulators; however, a link between changes in the auditory system and the UPR has not yet been reported, at least to the best of our knowledge. Similarly, few studies have reported the influence of metformin on ERK1/2 $(12,13)$, which directs various metabolic processes.

In this study, we investigated the molecular mechanisms of the UPR and AMPK/ERK1/2 pathway in the aging process and the effects of metformin on the process of age-related neurodegeneration. The findings of this study may provide a novel strategy for the protection of the brain against aging, specifically by treatment with metformin.

\section{Materials and methods}

Animal procedures. A total of 200 male Sprague-Dawley (SD) rats (weighing 80-100 g, 3 weeks old) were purchased from the Experimental Animal Center of Tongji Medical College, Huazhong University of Science and Technology. The rats were provided with free access to standard food and water and were housed in cages with an ambient temperature of $25^{\circ} \mathrm{C}$, a controlled humidity of $50 \%$ and a 12/12-h light/dark cycle. Prior to treatment, all rats were allowed to acclimatize to their environment for 1 week and were then randomly divided into 3 groups as follows ( $n=65-67$ per group): The control group, the D-galactose (D-gal) group and the D-gal + metformin group (Met group). The rats in the D-gal and Met groups were treated with D-gal (dissolved in $0.9 \%$ saline, $500 \mathrm{mg} / \mathrm{kg}$, Sigma) via subcutaneous injection for 8 weeks, while the rats in the control group were administered an equal volume of normal saline on the same schedule. The rats in the Met group were subjected to the daily administration of D-gal (subcutaneously) and metformin (intragastrically; metformin was dissolved in drinking water, $150 \mathrm{mg} / \mathrm{kg} / \mathrm{day}$, Dongrui Chemical Co.) for the same 8 weeks. After the 8 -week establishment of the model, the rats in the 3 groups were further randomly divided into the 3-month-old, 9-month-old (6 months post-injection) and 15-month-old (12 months post-injection) subgroups. Metformin was intragastrically administered to the rats in the Met groups (the 9-month-old Met group and the 15-month-old Met group) for 8 weeks before each sacrifice time point. The rats in the control and D-gal groups received the same dose of water without metformin on the same schedule. More detailed information on the animal procedures is provided in Fig. S1. All experimental procedures involving the care of animals were performed in strict accordance with the recommendations of the Guide for the Care and Use of Laboratory Animals of the National Institutes of Health (14). The protocol was under the supervision of the Committee on the Ethics of
Animal Experiments of Huazhong University of Science and Technology (Wuhan, China; permit no. IACUC S2219).

Auditory functions were examined by the auditory brainstem response (ABR) just before each sacrifice time point. When the rats were sacrificed, blood was harvested, and the brain tissues were immediately used in experiments or stored at $-80^{\circ} \mathrm{C}$ for later use.

Cell culture and treatment. Highly differentiated rat pheochromocytoma cells (PC12 cells) were obtained from the Ministry of Education Key Laboratory for Environment and Health of Tongji Medical College (HUST). The cells were cultured in RPMI-1640 medium with $10 \%$ fetal bovine serum (Gibco, Thermo Fisher Scientific) and 100 units/ml penicillin at $37^{\circ} \mathrm{C}$ in an incubator containing $5 \% \mathrm{CO}_{2}$ and humidified air. At $24 \mathrm{~h}$ after seeding, the cells were pre-treated with the ERK1/2 inhibitor, U0126 (Sigma), and the AMPK inhibitor, compound C (Sigma), for $30 \mathrm{~min}$. The cells were then treated with or without metformin (1,1-dimethylbiguanide hydrochloride, $100 \mu \mathrm{mol} / \mathrm{ml}$, Sigma) and cultured for $24 \mathrm{~h}$ as previously described (15), followed by incubation with D-gal $(15 \mathrm{mg} / \mathrm{ml})$ at $37^{\circ} \mathrm{C}$ for an additional $48 \mathrm{~h}$. Detailed information on the treatment of each group of PC12 cells is described in Fig. S2.

PC12 senescence model. PC12 cells were treated with increasing concentrations of D-gal $(3,6,9,12,15,18,21$ and $24 \mathrm{mg} / \mathrm{ml}$ ) for $48 \mathrm{~h}$; there was a significant decrease in cell number from $18 \mathrm{mg} / \mathrm{ml}$ (Fig. S3). The Cell Counting kit-8 (CCK-8) assay (Dojindo Laboratories) was used to determine the cell viability and cell proliferation according to the manufacturer's instructions and as previously described (16). D-gal was used to induce senescence in the PC12 cells, which were pre-treated with compound C (40 $\mu \mathrm{mol} / \mathrm{l})$, U0126 (10 $\mu \mathrm{mol} / \mathrm{l})$ or metformin $(100 \mu \mathrm{mol} / \mathrm{l})$ for $24 \mathrm{~h}$ for further experiments. The selected concentrations of the chemical inhibitors, D-gal and metformin did not affect cell viability (Fig. S3). The $\beta$-galactosidase ( $\beta$-Gal) assay, which measures the activity of $\beta$-Gal by immunohistochemistry, is a simple and effective method for measuring age-related changes in vitro and in vivo (17). The PC12 cells were stained using the $\beta$-Galactosidase Staining Kit (C0602, Beyotime) according to the manufacturer's instructions.

\section{Flow cytometry}

Annexin V/PI analysis. The Annexin V-FITC Apoptosis Detection kit (KeyGen Biotech) was used to detect cell apoptosis. The PC12 cells were harvested with EDTA-free $0.25 \%$ trypsin at $37^{\circ} \mathrm{C}$ in a cell incubator, washed twice with phosphate-buffered saline (PBS) and resuspended in $500 \mu \mathrm{l}$ of binding buffer. Subsequently, $5 \mu \mathrm{l}$ of Annexin V-FITC and PI were added to the $\mathrm{PC} 12$ cells for $15 \mathrm{~min}$ in the dark at room temperature and analyzed using a flow cytometer (Olympus).

Measurement of mitochondrial membrane potential $(\Delta \Psi m)$. A Mitochondrial Membrane Potential Assay kit with JC-1 (Beyotime) was used to detect changes in $\Delta \Psi_{\mathrm{m}}$ according to the manufacturer's instructoins. After each treatment, changes in the $\Delta \Psi \mathrm{m}$ of the PC12 cells were detected using the JC-1 assay. 
Measurement of ATP production. The ATP content in the cultured PC12 cells was determined using an adenosine 5'-triphosphate assay kit (A095, Nanjing) according to the manufacturer's instructions.

Hearing test: $A B R$. The auditory threshold was examined by evoking the ABR 4 times during the entire experiment (at 1, 3,9 and 15 months of age). The rats ( $n=10$ per group) were anesthetized with ketamine $(100 \mathrm{mg} / \mathrm{kg})$ and chlorpromazine $(5 \mathrm{mg} / \mathrm{kg}$ ) via intraperitoneal injection (18), placed in a double-walled radiofrequency and electrically shielded booth, and an insert earphone was set. Stainless steel needle electrodes were fixed subcutaneously ventrolateral to the right and left ears and the vertex for recording. The detailed procedures of the ABR test have been previously reported (1).

Morphological observation: Nissl staining analysis. The rat brains were fixed in $10 \%$ neutral formaldehyde solution, embedded in paraffin, and then sectioned at $5 \mu \mathrm{m}$. The sections were deparaffinized following standard procedures. The brain slices were soaked in $0.3 \%$ toluidine blue solution for $40 \mathrm{~min}$ at room temperature. The sections were then dehydrated using $95 \%$ ethanol for $5 \mathrm{sec}$, slightly counterstained with $0.5 \%$ eosin for $5 \mathrm{sec}$, cleared using xylene, placed under cover slips and analyzed under a light microscope (Leica).

Measurement of antioxidant enzyme activity. The levels of malondialdehyde (MDA), which results from lipid peroxidation and the activities of glutathione (GSH), superoxide dismutase (SOD) and catalase (CAT), were measured in vitro and in vivo using the appropriate kits (Nanjing Jiancheng Institute of Biological Engineering) according to the manufacturer's instructions and as previously described (19).

Western blot analysis. Tissues from both sides of the auditory cortex were dissected carefully on ice, and total protein was then extracted immediately from the tissues and from the PC12 cells with an ice-cold radioimmunoprecipitation assay (RIPA) lysis solution (Beyotime). The protein concentration of the samples from both the PC12 cells and the SD rats was quantified using a BCA Protein Assay kit (Beyotime). Equal amounts of protein from each sample were separated by $10-12 \%$ sodium dodecylsulfate polyacrylamide gel electrophoresis (SDS-PAGE) and electrotransferred onto polyvinylidene difluoride (PVDF) membranes. The membranes were blocked with $5 \%$ BSA for $1 \mathrm{~h}$ and incubated overnight at $4^{\circ} \mathrm{C}$ with an appropriate dilution of specific primary antibodies. The following antibodies were used: Anti-extracellular signal-regulated kinase (ERK)1/2 and anti-p-ERK1/2 (diluted 1:1,000, 4695P and 4370P, Cell Signaling Technology); anti-forkhead transcription factor (Foxo)3 (diluted 1:1,000, NBP2-24579, Novus Biologicals); anti-p-Foxo3 (diluted 1:1,000, ARG51655, Arigo Biolaboratories); anti-HSP90 $\alpha$ (diluted 1:2,000, GTX109753, GeneTex); anti-HSP60 (diluted 1:1,000, 4870, Sigma); anti-AMPK $\alpha 1 / 2$ (diluted 1:1,000, 21191, Signalway); anti-p-AMPK $\alpha 1 / 2$ (diluted 1:600, sc-33524, Santa Cruz Biotechnology); anti-GRP78 (diluted 1:2,000, GTX102580, GeneTex); anti-GADD153/CHOP (diluted 1:3,000, NBP2-13172, Novus Biologicals); anti-caspase-3 and anti-cleaved caspase-3 (diluted 1:1,000, 9662 and 9661, Cell
Signaling Technology); anti-p53 (1:200, sc-98, Santa Cruz Biotechnology); anti- $\alpha$-tubulin (diluted 1:1,000, ab18251, Abcam) and anti- $\beta$-actin (diluted 1:3,000, Mab1445, Lianke). The membranes were washed 4 times before being incubated with appropriate horseradish peroxidase (HRP)-conjugated secondary antibodies (diluted 1:4,000, ANT019 and ANT020, AntGene) for $1 \mathrm{~h}$ at room temperature and developed using ECL Plus reagents (Beyotime) to visualize the membranes. Protein quantification was performed using Quantity One 4.6.2 Software (Bio-Rad).

Quantitative polymerase chain reaction ( $q P C R$ ). Total DNA was extracted from the tissues from both sides of the auditory cortex and from $10^{7}$ cultured neurons using a Genomic DNA Purification kit (Tiangen Biotech Co., Ltd.). The purification and concentration of the DNA was evaluated with a Gene Quant Pro DNA/RNA Calculator (BioChrom). Primers and probes specifically designed for mitochondrial DNA (mtDNA) CD and mtDNA D-loop have been described previously (20). The LC-480 Real-time PCR system (Roche Diagnostics Ltd.) was used for performing the PCR amplification. TaqMan PCR mix (2X, $10 \mu 1$, Takara), a probe $(0.2 \mu \mathrm{l}$ for each, $10 \mathrm{mM})$, primers $(0.4 \mu \mathrm{l}$ of each reverse and forward, $10 \mathrm{mM})$, distilled water (5 $\mu \mathrm{l})$, and sample DNA (4 $\mu 1,10 \mathrm{ng} / \mathrm{ml}$ ) comprised the $20-\mu 1$ reaction mixture. The cycling conditions were as follows: An initial phase at $95^{\circ} \mathrm{C}$ for $30 \mathrm{sec}$, then 40 cycles at $95^{\circ} \mathrm{C}$ for 5 and $30 \mathrm{sec}$ at $60^{\circ} \mathrm{C}$. $\triangle \mathrm{Cq}\left(\mathrm{Cq}_{\text {deletion }}-\mathrm{Cq}_{\mathrm{D} \text {-loop }}\right)$ was used to calculate the abundance of the mtDNA $4,834 \mathrm{bp}$ deletion. The relative expression indicates the factorial difference in the deletions between the subgroups, and it was calculated using the $2^{-\Delta \Delta \mathrm{Cq}}$ method (21), where $\Delta \Delta \mathrm{Cq}=\Delta \mathrm{Cq}_{\mathrm{mtDNA}}$ deletion in D-gal group $-\Delta \mathrm{Cq}_{\mathrm{mtDNA} \text { deletion in }}$ control group or $\Delta \Delta \mathrm{Cq}=\Delta \mathrm{Cq}_{\mathrm{mtDNA} \text { deletion in Met group }}-\Delta \mathrm{Cq}_{\mathrm{mtDNA} \text { deletion in }}$ D-gal group. $\mathrm{qPCR}$ and the detailed method of calculation were performed as reported previously by our laboratory (22).

Electron microscopy. Transmission electron microscopy (TEM) was used to examine the ultrastructure of the auditory cortex and the cultured PC12 cells. After the PC12 cells were treated with the appropriate drugs, the cells were fixed in $2.5 \%$ glutaraldehyde (Sigma) at $4^{\circ} \mathrm{C}$ for $24 \mathrm{~h}$. A total of $27(\mathrm{n}=3$ from each subgroup) were used for the TEM assay. The extraction of the specimens and the preparation, conditions, and procedures were performed as previously described $(22,23)$.

TUNEL assay. A TUNEL Apoptosis Detection kit (FITC; Yeasen) was used to detect PC12 cell apoptosis. At the same time, the nuclei were counterstained with a DAPI staining solution ( $1 \mu \mathrm{g} / \mathrm{ml}$; Beyotime) at room temperature for 3-5 min. A laser-scanning confocal microscope (Nikon) was used to examine the cells.

Statistical analysis. The data are expressed as the means \pm standard deviation (SD). Statistical analyses were conducted using GraphPad Prism 5.0 software and Microsoft Excel. A two-tailed, unpaired Student's t-test were used to determine statistical significance when comparing 2 groups, and one-way analysis of variance (ANOVA) followed by a Dunnett's multiple comparisons test was used to compare differences between $>2$ groups. Differences with a P-value $<0.05$ were 


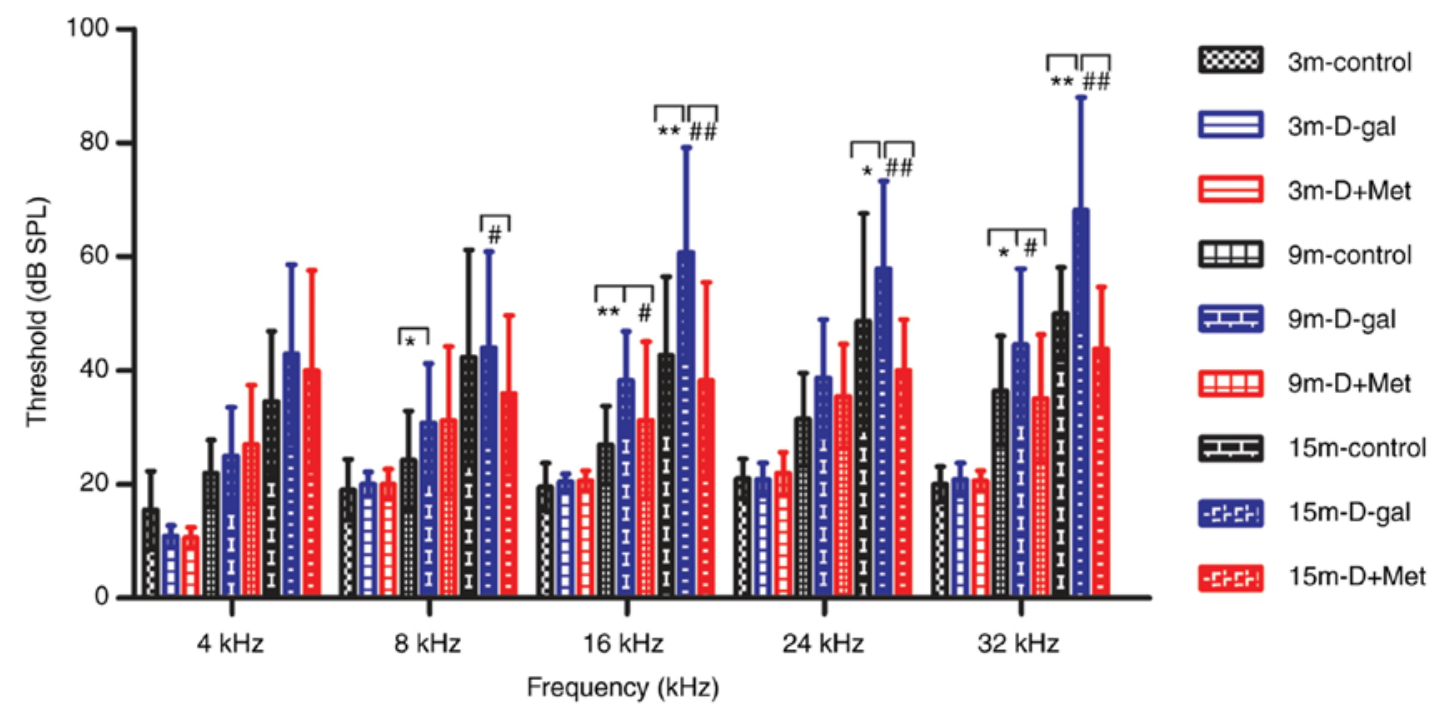

Figure 1. ABR thresholds in the different groups of rats. The data are expressed as the means \pm SD of 10 rats per group. ${ }^{*} \mathrm{P}<0.05,{ }^{, * *} \mathrm{P}<0.01 ;{ }^{*} \mathrm{P}<0.05,{ }^{\# \#} \mathrm{P}<0.01$. ABR, auditory brainstem response; $\mathrm{dB}$ SPL, decibels of sound pressure level; m, months; D-gal, D-galactose; Met, metformin.

considered to indicate statistically significant differences. All experiments were repeated independently at least 3 times.

\section{Results}

Metformin treatment alleviates age-related hearing loss. The ABR threshold was significantly elevated in the 15-month-old rats compared with the 3 -month-old rats. There was a significant increase in the average ABR threshold in the D-gal group compared with the control group, while metformin significantly suppressed this elevation, particularly in the 15-month-old rats (Fig. 1). This finding indicated that D-gal accelerated AHL and that metformin exerted a protective effect on D-gal-induced aging in rats.

Metformin attenuates the neurodegeneration induced by $D$-gal in vivo and in vitro. TEM was used to examine the effects of metformin on neurodegeneration at the ultrastructural level in the auditory cortex and in cultured PC12 cells. The sections from the PC12 cells were analyzed at $\mathrm{x} 1,700$ and $\mathrm{x} 5,000$ magnification (Fig. 4). The sections from the auditory cortex were analyzed at x1,500, x5,000, and x6,800 magnification, respectively focusing on the nuclei, organelles and the myelin. In all rats in the 3-month-old groups (Fig. 2A-C), the nuclear membrane was intact, and the nuclei $(\mathrm{N})$ were round in shape. The chromatin in the 9-month-old control group (Fig. 2D) was uniform, and the mitochondria (white arrows) were normal. In the 9-month-old D-gal group (Fig. 2E), some irregular nuclei and condensed chromatin were noted; moreover, these changes were more significant in the rats in the 15-month-old D-gal group (Fig. 2H). Visible ultrastructural degeneration, such as condensed chromatin, vacuolated mitochondria (black arrows), and irregular nuclei, were found in the 15-month-old rats (Fig. 2G-I). Additionally, the myelin was swollen and disrupted (white arrowhead), and lipofuscin was abundant (black arrowhead). Metformin treatment alleviated these changes induced by D-gal, as the ultrastructure of the organelles appeared healthier (Fig. 2C, F and I).
In addition, $\beta$-galactosidase staining was performed to detect the apoptosis of $\mathrm{PC} 12$ cells. A significant accumulation of positively stained cells was observed in the D-gal $(15 \mathrm{mg} / \mathrm{ml})$ group, while pre-treatment with metformin reduced the number of positive cells (Fig. 3A and B). Considering these results, our model of senescent $\mathrm{PC} 12$ cells, which was induced

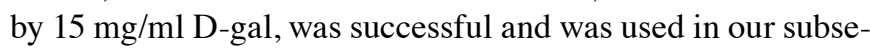
quent experiments.

In our in vitro experiments, abundant normal mitochondria (white arrows), an intact nuclear membrane and normal-shaped nuclei were observed in the PC12 cells. However, in the D-gal group, the mitochondria were swollen and vacuolated (white arrows); however, pre-treatment with metformin alleviated the mitochondrial damage. However, the inhibition of ERK1/2 and AMPK aggravated these changes (Fig. 4).

Toluidine blue staining was used to determine neuronal loss in the auditory cortex region, the sections were analyzed at x200 and x400 magnification (Fig. 5). The results demonstrated that the number of neurons in the auditory cortex regions was significantly reduced in the D-gal group compared with the control group, and these alterations were significantly attenuated by metformin treatment. In the rats in the same age groups, the rats in the control group and Met group had numerous neurons compared with those in the D-gal group, and the neurons in the rats in the control group and Met group were arranged tightly and were morphologically intact. An irregular structure, a decreased number of pyramidal cells, and degenerated pyramidal cells were visible in the auditory cortex region of the brains of 15-month-old rats (Fig. 5A and B).

Metformin alleviates the diminished function of the antioxidant system via the AMPK/ERK1/2 signaling pathways. We examined the activity of antioxidants and investigated the mechanisms underlying the effects of metformin on antioxidants. The activities of the antioxidants, GSH, SOD and CAT, were decreased in the blood serum of the rats in the 3-, 9- and 15-month-old D-gal groups, respectively, compared with those in the age-matched control groups, while concurrent metformin 

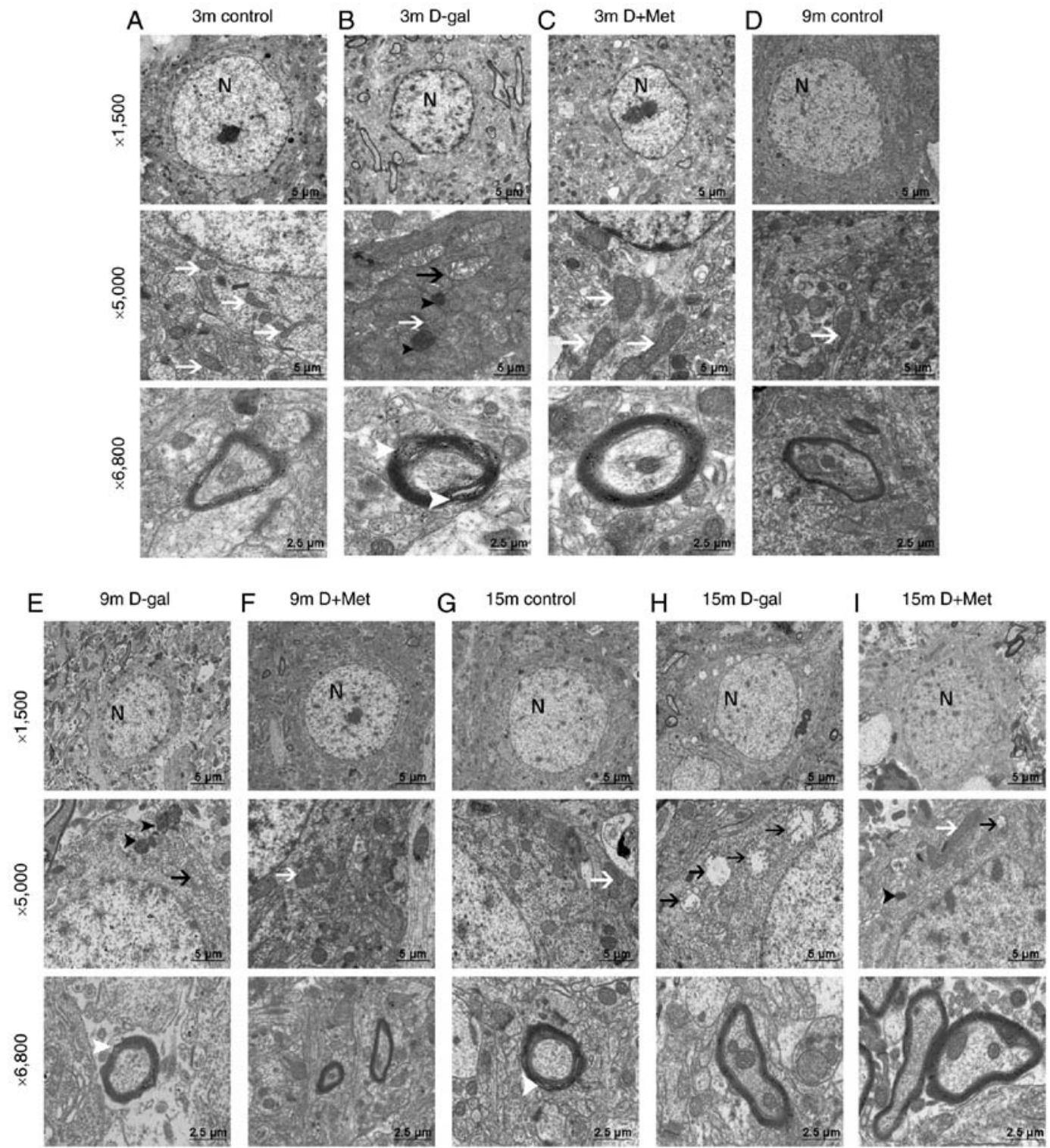

Figure 2. (A-I) Ultrastructural morphology of the auditory cortex. The nuclei (N) and the mitochondria (white arrows) were normal in the control groups. Vacuolated mitochondria (black arrows), and irregular nuclei were found in the D-gal groups. The myelin was swollen and disrupted (white arrowhead), and lipofuscin was abundant (black arrowheads) in the 15-month-old rats. Metformin attenuated the D-gal-induced neurodegeneration in the auditory cortex at different ages. m, months; D-gal, D-galactose; Met, metformin.

treatment significantly attenuated this decrease (Fig. 6A-C). In addition, we also found that the levels of CAT, GSH and SOD were reduced with age. These alterations in enzyme activity were also detected in the PC12 cells, suggesting that GSH, SOD and CAT enzyme activities were markedly decreased in the senescent aging model and increased upon treatment with metformin (Fig. 6E-G). MDA is a product of lipid peroxidation mediated by oxygen free radicals and is widely known as a marker of oxidative stress (24). The data indicated that the MDA levels in the D-gal group increased significantly compared with those in the age-matched control group. In the metformin group, the MDA level was decreased compared with the age-matched D-gal group. In addition, the levels of MDA increased with aging (Fig. 6D and H). All these changes caused by D-gal were attenuated by metformin treatment, while the effect was partially suppressed when the PC12 cells were treated with the AMPK and ERK1/2 inhibi- tors (Fig. 6E-H). The results also implied that the improved antioxidant capacity induced by metformin occurred through the AMPK and ERK1/2 signaling pathways to a certain extent.

Metformin protects the mitochondria through the AMPK/ERK1/2 pathways. The function of the mitochondria was determined through the evaluation of mitochondrial membrane permeabilization and ATP production. The flow cytometry data revealed a decreased mitochondrial membrane permeabilization in the D-gal group, which indicated that the number of early apoptotic mitochondria was increased (Fig. 7B and D). Annexin V/PI staining and flow cytometry analysis of cell death were repeated 4 times and similar results were obtained (Fig. 7A). The average values of the percentages of apoptotic cells from repeated experiments are shown in Fig. 7C. The number of apoptotic mitochondria significantly decreased with metformin treatment, while the protective 
A

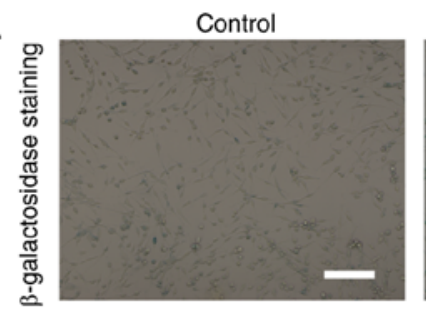

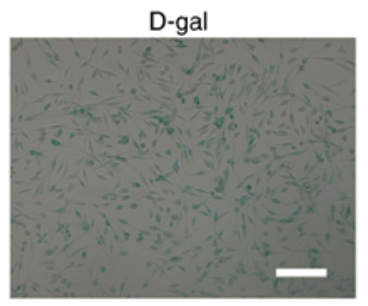

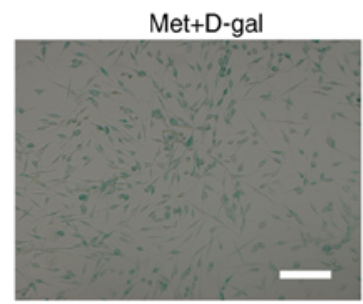

B

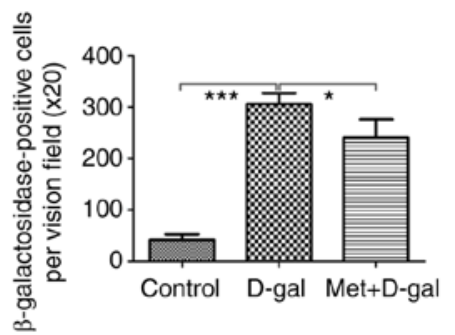

C

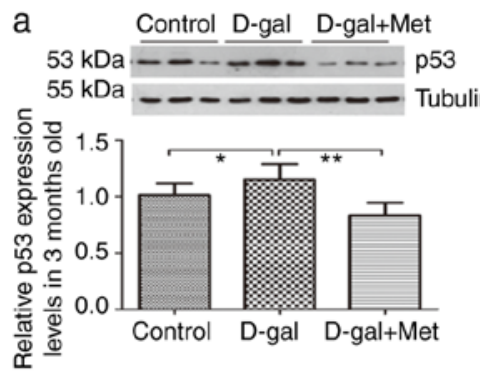

d

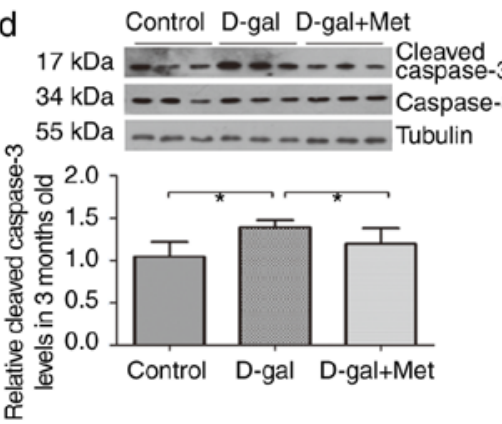

b

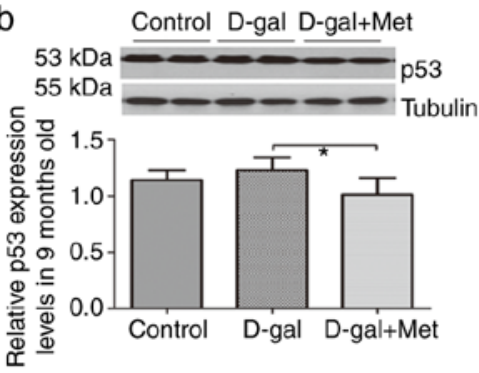

e

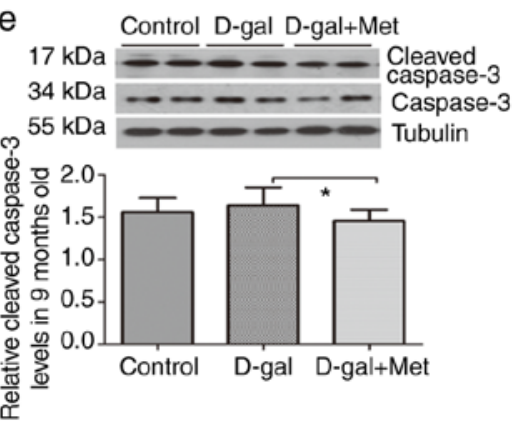

C
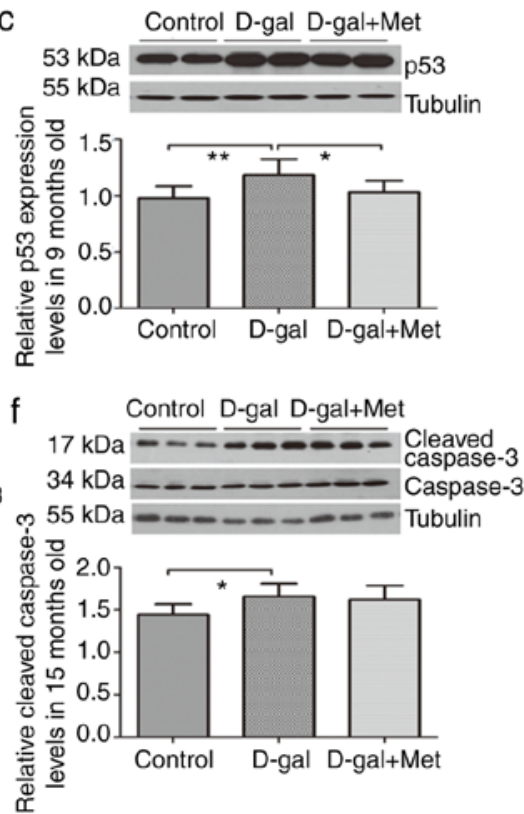

Figure 3. Determination of senescence in PC12 cells. (A) Senescence was evaluated in PC12 cells treated with $15 \mathrm{mg} / \mathrm{ml}$ of D-gal by the Senescence $\beta$-Galactosidase Staining kit. Scale bar, $25 \mu \mathrm{m}$. (B) Cells positive for $\beta$-galactosidase staining were counted and are shown in panel, $\mathrm{n}=6$ for each subgroup. (C) Western blot analysis of (a-c) p53 and (d-f) caspase-3 expression in the auditory cortex of rats. ${ }^{*} \mathrm{P}<0.05,{ }^{* *} \mathrm{P}<0.01,{ }^{* * *} \mathrm{P}<0.001$. The data are presented as the means $\pm \mathrm{SD} ; \mathrm{n}=6$ for each subgroup. D-gal, D-galactose; Met, metformin.

effect was suppressed when the PC12 cells were treated with AMPK and ERK1/2 inhibitors (Fig. 7). In addition, we detected the ATP levels in the PC12 cells. ATP, which is produced by mitochondria, is the most intuitive indicator of MT function. The levels of ATP decreased in the D-gal group, but increased with metformin treatment; however, when the PC12 cells were simultaneously treated with AMPK or ERK1/2 inhibitors, metformin could not increase the ATP levels (Fig. 7E). Taken together, these results indicated that metformin protected the mitochondria through the AMPK and ERK1/2 signaling pathways to a certain extent.

Metformin treatment decreased the accumulation of the mtDNA common deletion (CD) in vitro and in vivo (Fig. 5C and D). Previous studies have confirmed that the mitochondrial 4,834-bp deletion is a biomarker of AHL, and the 4,834-bp deletion gradually deteriorates with age $(25,26)$. In this study, compared with age-matched control groups, the D-gal groups exhibited a markedly elevated CD level (Fig. 5C); CD accumulated with age, and metformin decreased the CD level throughout the process. The same phenomenon was observed in the PC12 cells (Fig. 5D).

\section{Metformin prevents apoptosis via the AMPK/ERK1/2 signaling} pathways. To investigate the stress-related apoptotic factors, caspase-3 and p53, and to examine the effects of metformin on these proteins, changes in protein expression were examined by western blot analysis. In the auditory cortex tissues, significant increases in p53 and cleaved caspase-3 expression were noted after the injection of D-gal. However, we found a significantly decreased expression of p53 and cleaved caspase- 3 following treatment with metformin (Fig. 3C). We also analyzed apoptosis by counting the number of cells stained by TUNEL assay. Apoptosis Detection kit (FITC) staining and Annexin V/PI staining were performed on the PC12 cells. The right upper 

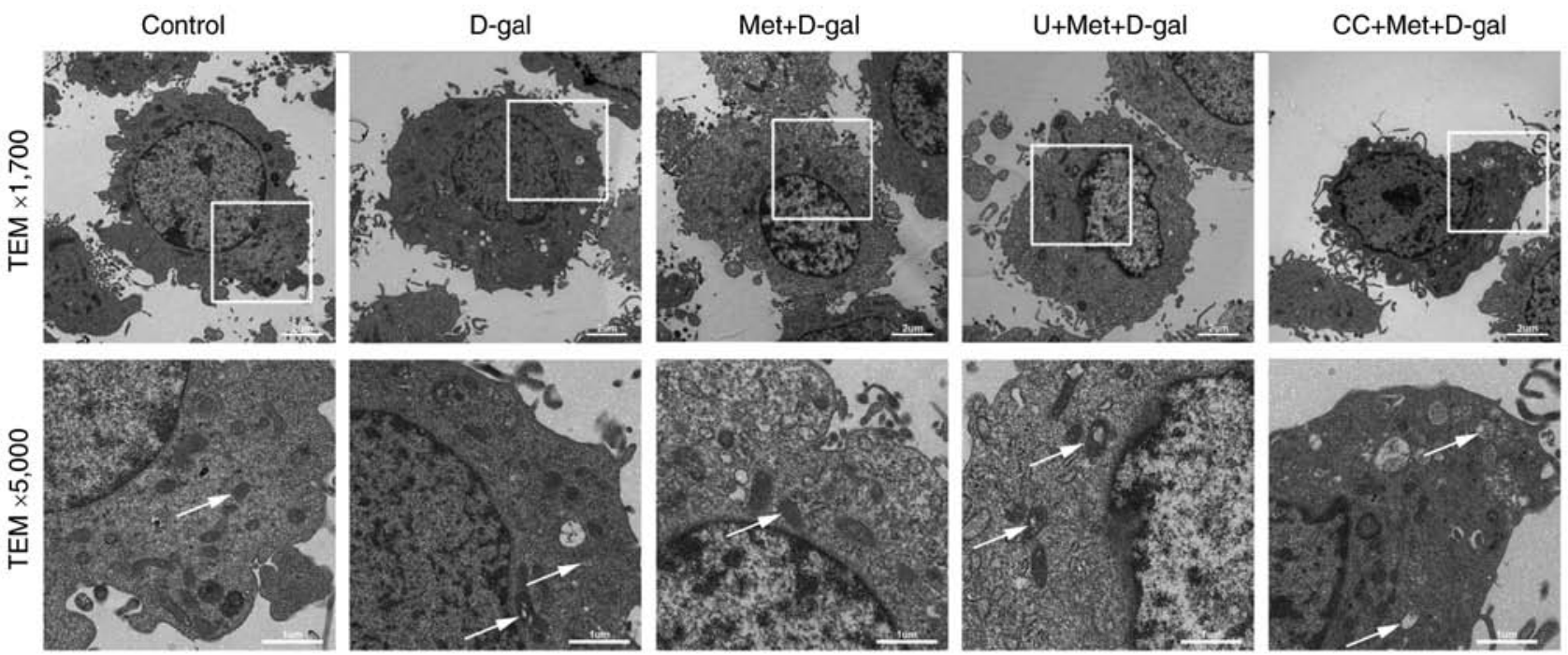

Figure 4. Electron photomicrographs of PC12 cells. Abundant normal mitochondria (white arrows), an intact nuclear membrane, and normal-shaped nuclei were found in PC12 cells. However, the mitochondria were swollen and vacuolated in the D-gal group, and pretreatment with metformin alleviated the mitochondrial damage. The images on the bottom panels are a larger magnification of the area depicted in the white boxes on the top panels. D-gal, D-galactose; Met, metformin.
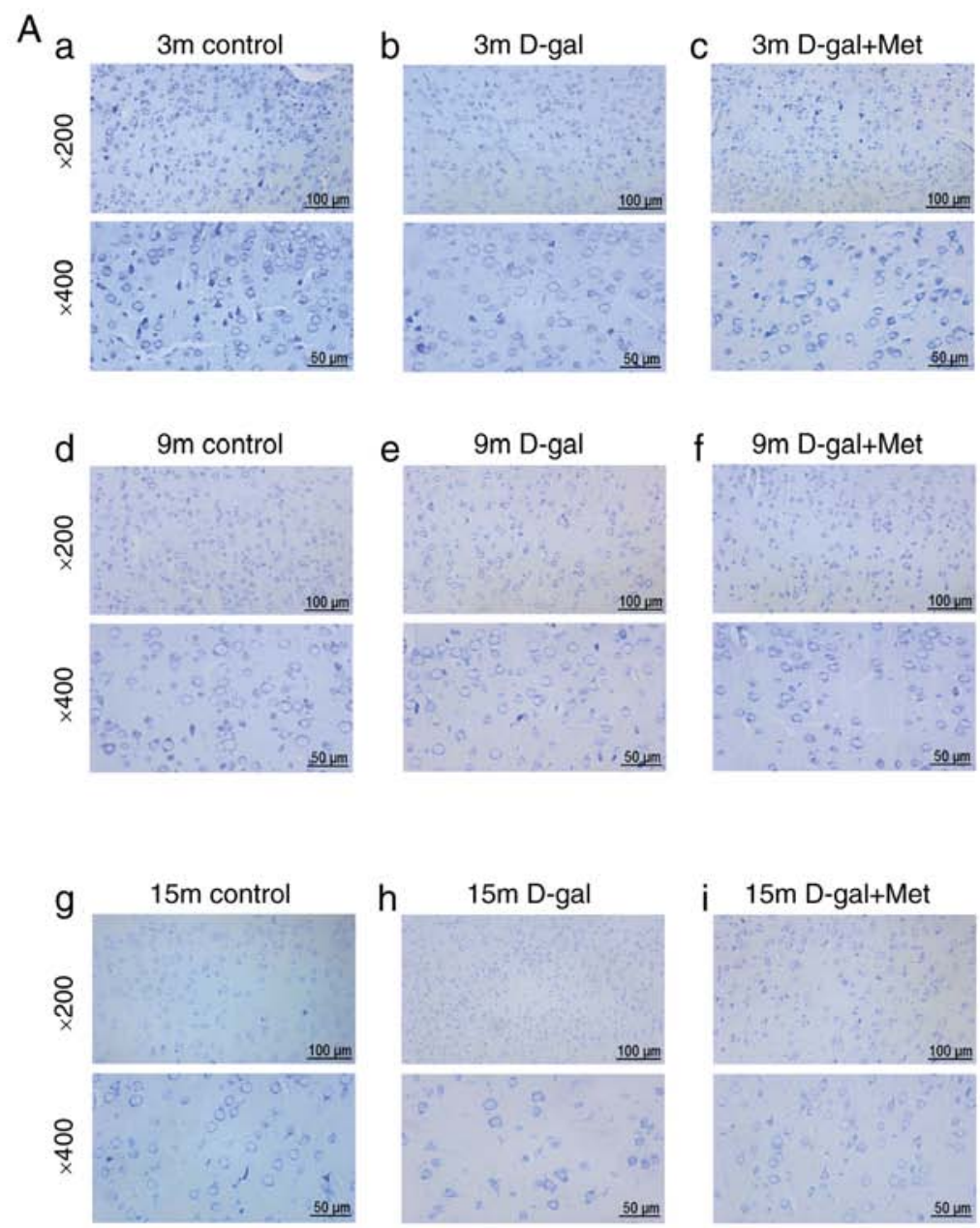

\section{B}
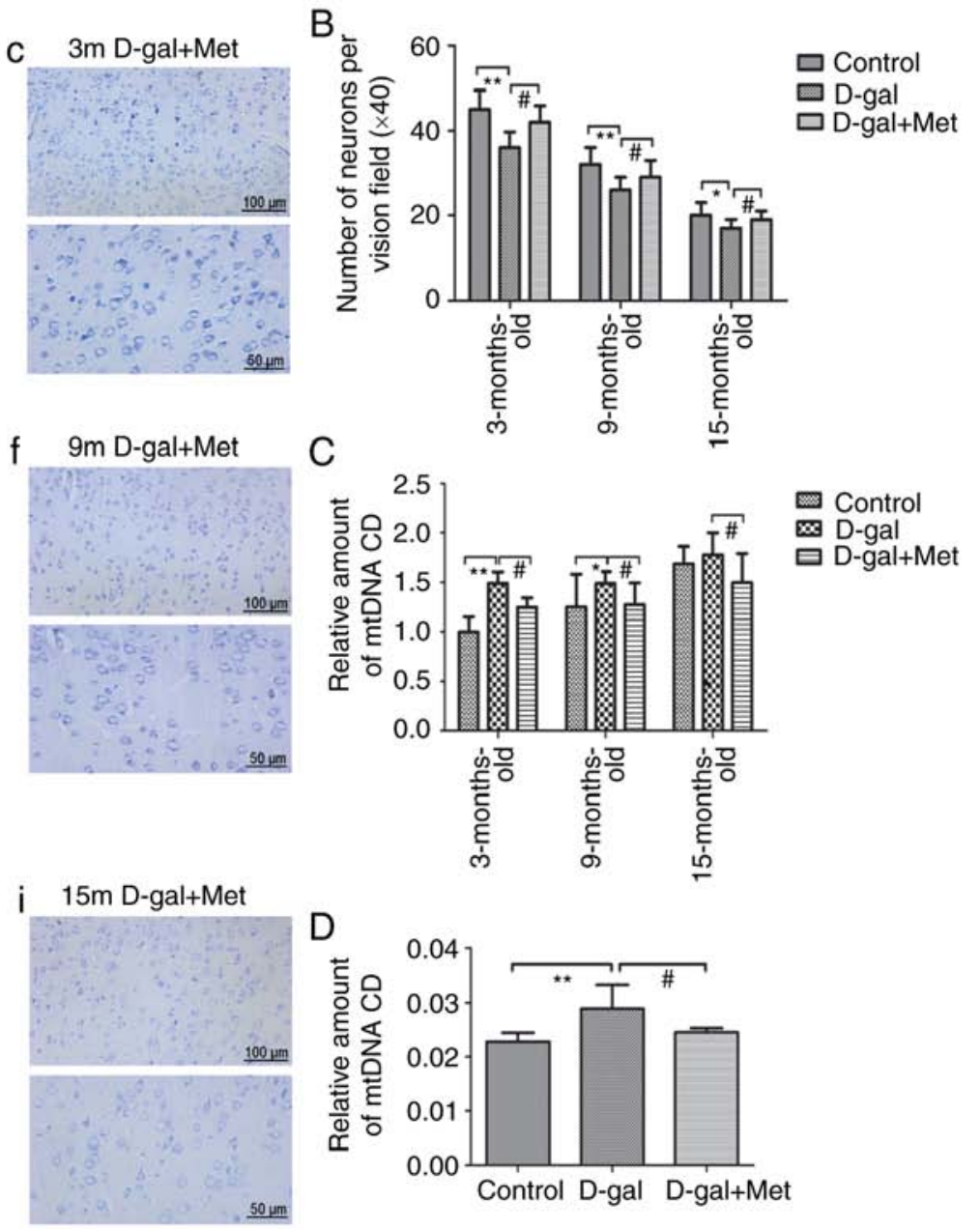

Figure 5. (A) Sections from the auditory cortex of rats were stained with toluidine blue and used to count surviving neurons. The sections from the auditory cortex were analyzed at x200 and x400 magnification, (a-c) 3-Month-old rats; (d-f) 9-month-old rats; (g-i) 15-month-old rats. The top images were analyzed at x200 magnification, and the bottom images were analyzed at x400 magnification ( $\mathrm{n}=6$ for each subgroup); each paraffin-embedded rat brain sample was cut into several slices; the images in the top and bottom panels are the same mouse brain, but from different slices. (B) The corresponding statistical chart is displayed. (C) qPCR analysis of CD 4,834 levels in the 3-, 9-, and 15-month-old control, D-gal and Met groups. (D) qPCR analysis of CD 4,834 levels in PC12 cells. ${ }^{*} \mathrm{P}<0.05,{ }^{* *} \mathrm{P}<0.01 ;{ }^{*} \mathrm{P}<0.05$. The data are displayed as the means $\pm \mathrm{SD} ; \mathrm{n}=6$ for each subgroup. $\mathrm{m}$, months; D-gal, D-galactose; Met, metformin; $\mathrm{CD}$, common deletion. 

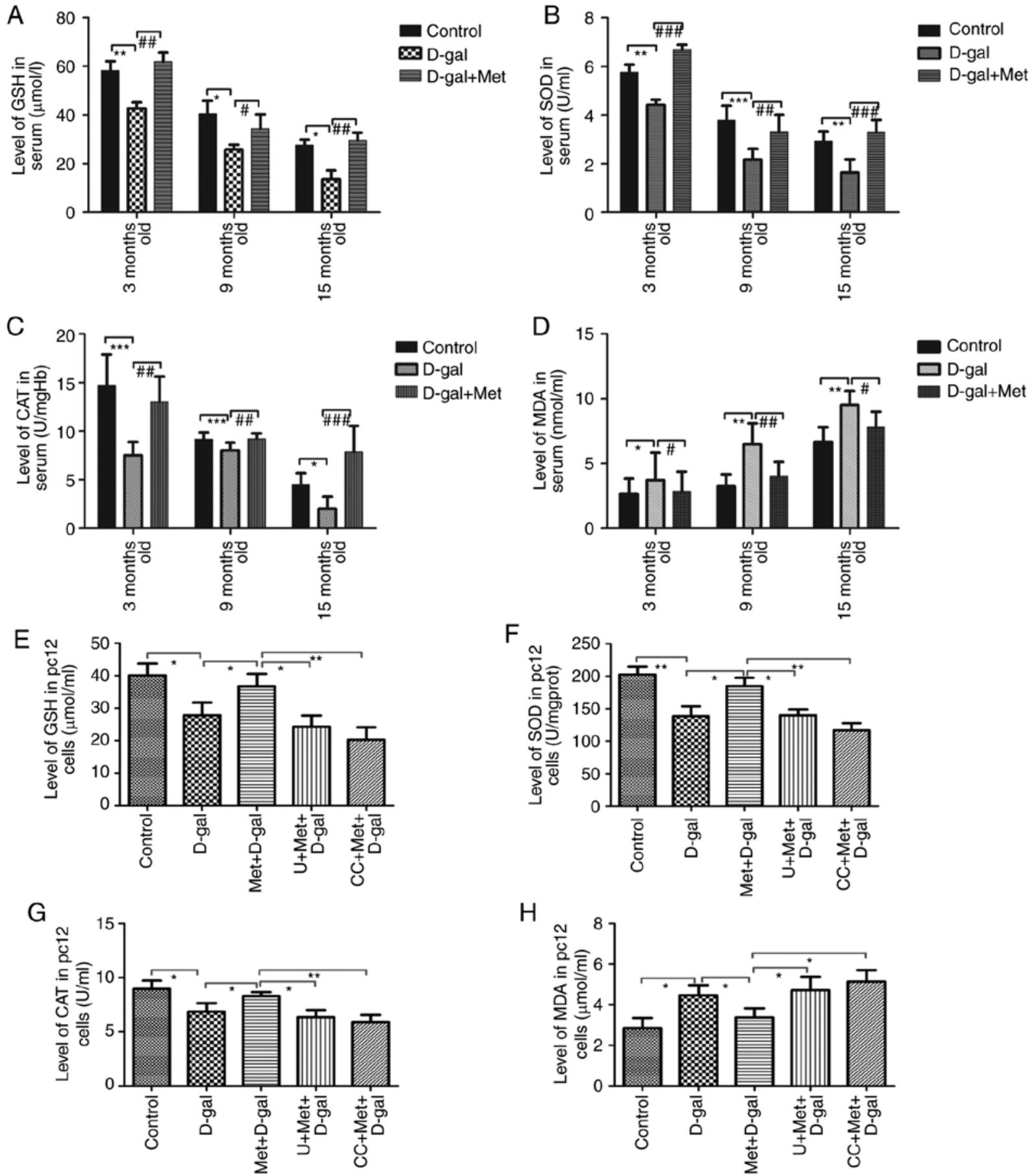

Figure 6. Analysis of antioxidants in vitro and in vivo. Metformin treatment significantly increased the (A) GSH, (B) SOD and (C) CAT levels in the auditory cortex of D-gal-injected rats. Metformin treatment significantly decreased the (D) MDA level compared with that in the respective D-gal groups. The activities of these enzymes were detected in PC12 cells, suggesting that (E) GSH, (F) SOD, (G) CAT and (H) MDA enzyme activities were markedly decreased in the senescent aging model and increased upon treatment with metformin; however, this upregulation was prevented significantly by inhibitors of AMPK and ERK1/2. The data are presented as the means $\pm \mathrm{SD}(\mathrm{n}=6) .{ }^{*} \mathrm{P}<0.05,{ }^{* *} \mathrm{P}<0.01,{ }^{* * *} \mathrm{P}<0.001,{ }^{\#} \mathrm{P}<0.05,{ }^{\# \#} \mathrm{P}<0.01,{ }^{\# \# \#} \mathrm{P}<0.001$. D-gal, D-galactose; Met, metformin; SOD, superoxide dismutase; GSH, glutathione; MDA, malondialdehyde.

quadrant and right lower quadrant represent the late and early apoptotic cells, respectively. The number of apoptotic cells in the Q2 and Q3 panels (upper and lower right) was calculated, and the percentages of cells undergoing apoptosis was determined (Fig. 7A). Significant increases in the number of TUNEL-positive cells were noted after the injection of D-gal; however, treatment with metformin decreased the percentage of apoptotic cells (Fig. 8). Moreover, treatment with AMPK and ERK1/2 inhibitors partially reversed the protective effects of metformin. Thus, metformin alleviated the apoptosis of the PC12 cells through the AMPK and ERK1/2 pathways.

Metformin regulates the UPR to maintain protein homeostasis in vivo and in vitro. Protein misfolding and aggregation are common pathological features of a number of neurodegenerative diseases (27). In this study, significant increases in the 


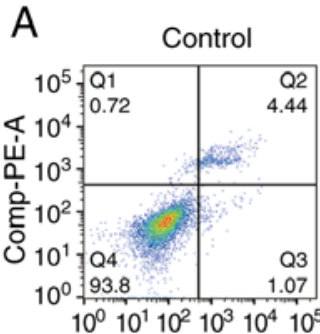

Comp-FITC-A
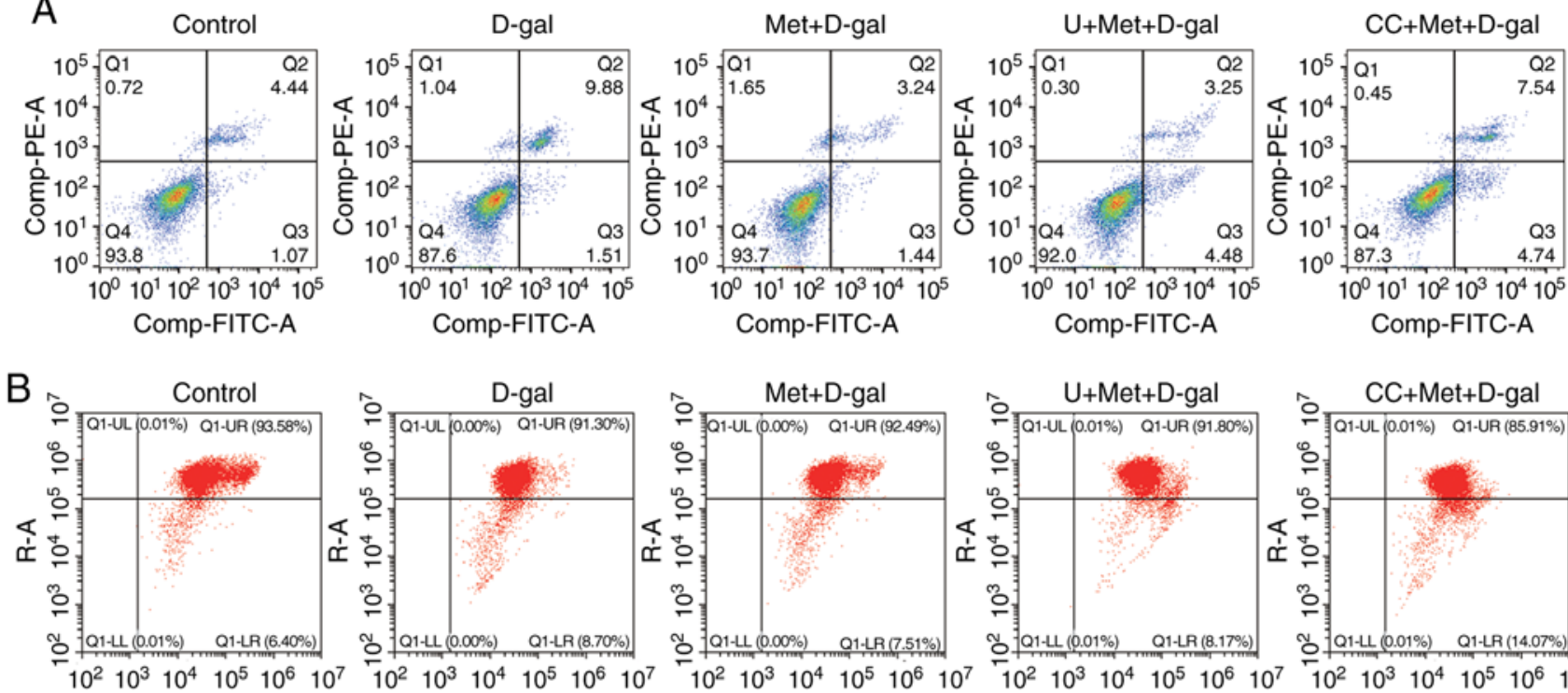

G-A

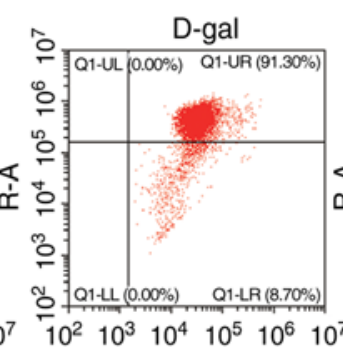

G-A
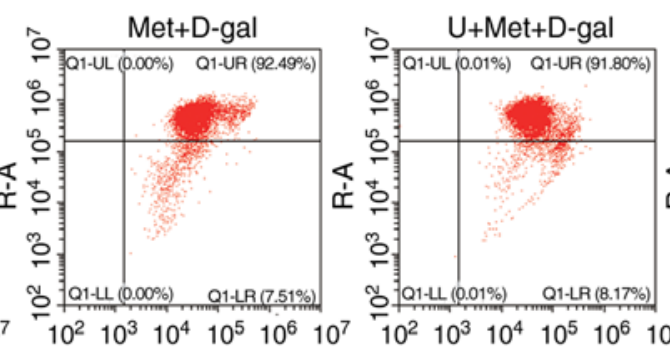

G-A

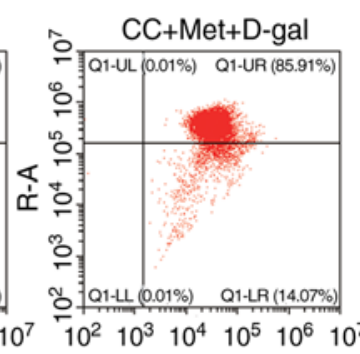

G-A

E
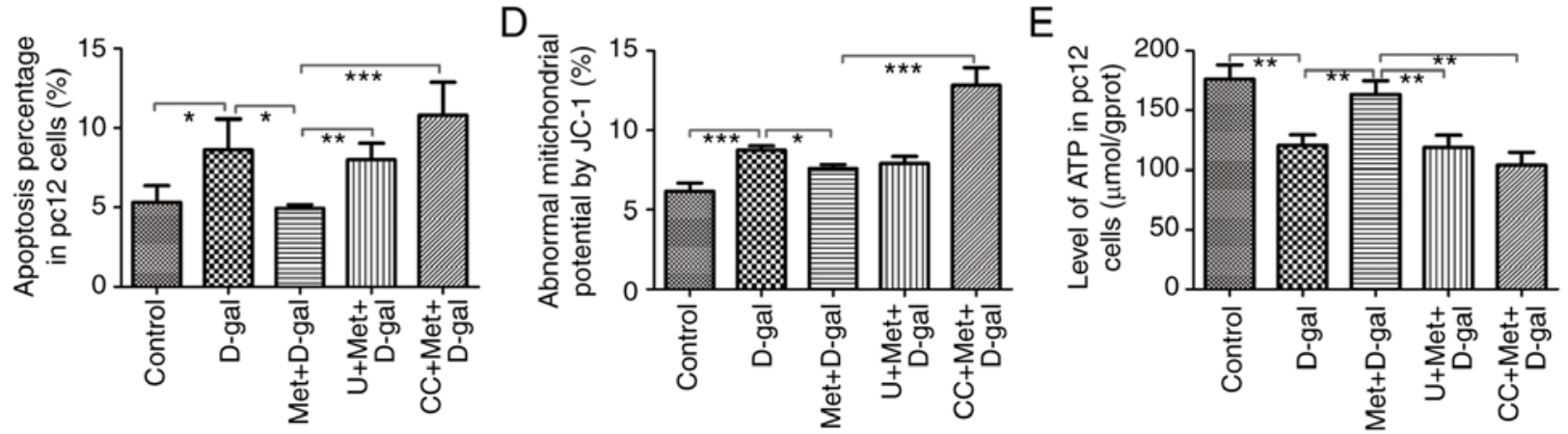

Figure 7. (A) Annexin V/PI staining and flow cytometry analysis of cell death. LL (lower left) indicates viable PC12 cells. UR (upper right) and LR (low right) represent the late apoptotic cells and early apoptotic cells respectively. UR and LR panels were summed as the total percentage of apoptotic cells in this experiment. This experiment was repeated four times and similar results were obtained. (C) The average value of the percentages of apoptotic cells from repeated experiments are shown. (B) Following treatment, the cells were stained with JC-1 and analyzed by flow cytometry. A decreased mitochondrial membrane permeabilization is a hallmark of early apoptosis. (D) D-gal increased the expression level of abnormal mitochondrial membrane potential, and pre-treatment with metformin reduced it. (E) ATP production in PC12 cells from each group was determined. ${ }^{*} \mathrm{P}<0.05,{ }^{* * *} \mathrm{P}<0.01,{ }^{* * * *} \mathrm{P}<0.001$. The data are presented as the means $\pm \mathrm{SD} ; \mathrm{n}=6$ for each subgroup. D-gal, D-galactose; Met, metformin; U, U0126; CC, compound C.

levels of UPR-related proteins were observed after the injection of D-gal, which is consistent with the findings of previous studies $(28,29)$. The ER stress markers, GRP78 and CHOP, are also known as major modulators of the ER-UPR. HSP90 and HSP60 are chaperone proteins that assist other proteins in folding properly and are also modulators of the MT-UPR. In our in vivo experiments, we found that the expression levels of HSP90, HSP60, GRP78 and CHOP were upregulated in the 3-month-old D-gal groups; however, metformin significantly decreased these expression levels. The levels of the MT-UPR chaperone proteins, HSP90 and HSP60, were still upregulated in the 15-month-old D-gal groups, and metformin significantly decreased these expression levels. However, the expression levels of the ER-UPR chaperone proteins, GRP78 and CHOP, decreased in the 15-month-old D-gal group compared with the control group, but increased in the 15-month-old Met group compared with the D-gal group (Fig. 9). In our in vitro experiments, HSP90 expression was upregulated following treatment with D-gal, and its expression increased further following additional treatment with metformin. Treatment with AMPK and ERK1/2 inhibitors significantly reversed the effects of metformin (Fig. 10C). A significant elevation in GRP78 expression was also observed in the $\mathrm{PC} 12$ cells following treatment with D-gal; however, treatment with metformin led to a significant decline in GRP78 expression. Conversely, in the groups pre-treated with U0126, metformin did not decrease GRP78 expression, but when the groups were pre-treated with compound C, changes were not evident (Fig. 10D). These findings focus on the UPR pathways, suggesting the role of metformin in the maintenance of cellular homeostasis.

Metformin regulates the AMPK/ERK1/2 signaling pathways in vivo and in vitro during aging. AMPK and ERK1/2 act independently, and the crosstalk between them is frequent. Notably, the crosstalk is highly variable; both cross-inhibition and cross-activation can occur depending on the cell type (30). In this study, metformin treatment significantly activated p-AMPK and p-Foxo3 $\alpha$ in the 3-, 9-, and 15-month-old rats (Fig. 11A and B). A significant decrease in the phosphorylation levels of Foxo3 $\alpha$ and ERK1/2 was noted after the injection of D-gal. By contrast, we observed an increase in the Foxo3 $\alpha$ and ERK1/2 phosphorylation levels in the 3-month-old metformin group (Fig. 11B and C); 

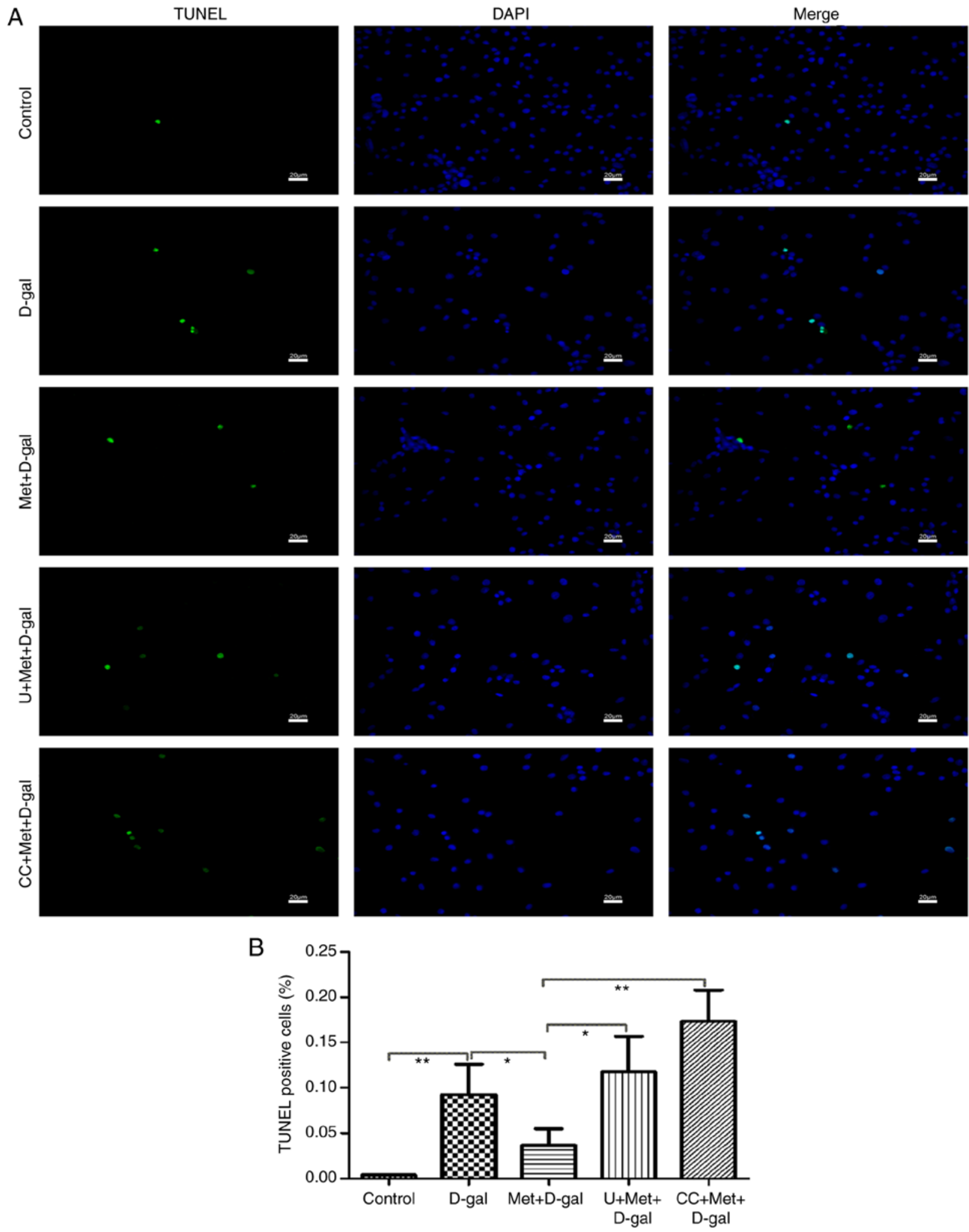

Figure 8. (A and B) The number of apoptotic cells was determined by TUNEL staining in PC12 cells. ${ }^{*} \mathrm{P}<0.05,{ }^{* *} \mathrm{P}<0.01$. The data are presented as the means $\pm \mathrm{SD} ; \mathrm{n}=6$ for each subgroup. D-gal, D-galactose; Met, metformin; U, U0126; CC, compound C.

however, the ERK1/2 phosphorylation levels were decreased in the 15-month-old metformin group (Fig. 11C). In our in vitro experiment, a significant elevation in the phosphorylation levels of ERK1/2 and AMPK was noted following treatment with D-gal, and a further elevation in the levels of these proteins was found following co-treatment with metformin. In addition, the inhibition of ERK1/2 blocked the effects mediated by metformin. However, surprisingly, the inhibition of AMPK slightly increased the phosphorylation level of ERK1/2. Thus, there was some association between ERK1/2 and AMPK, indicating that the AMPK pathway can inhibit the ERK1/2 pathway in PC12 cells to a certain extent (Fig. 10A and B). 

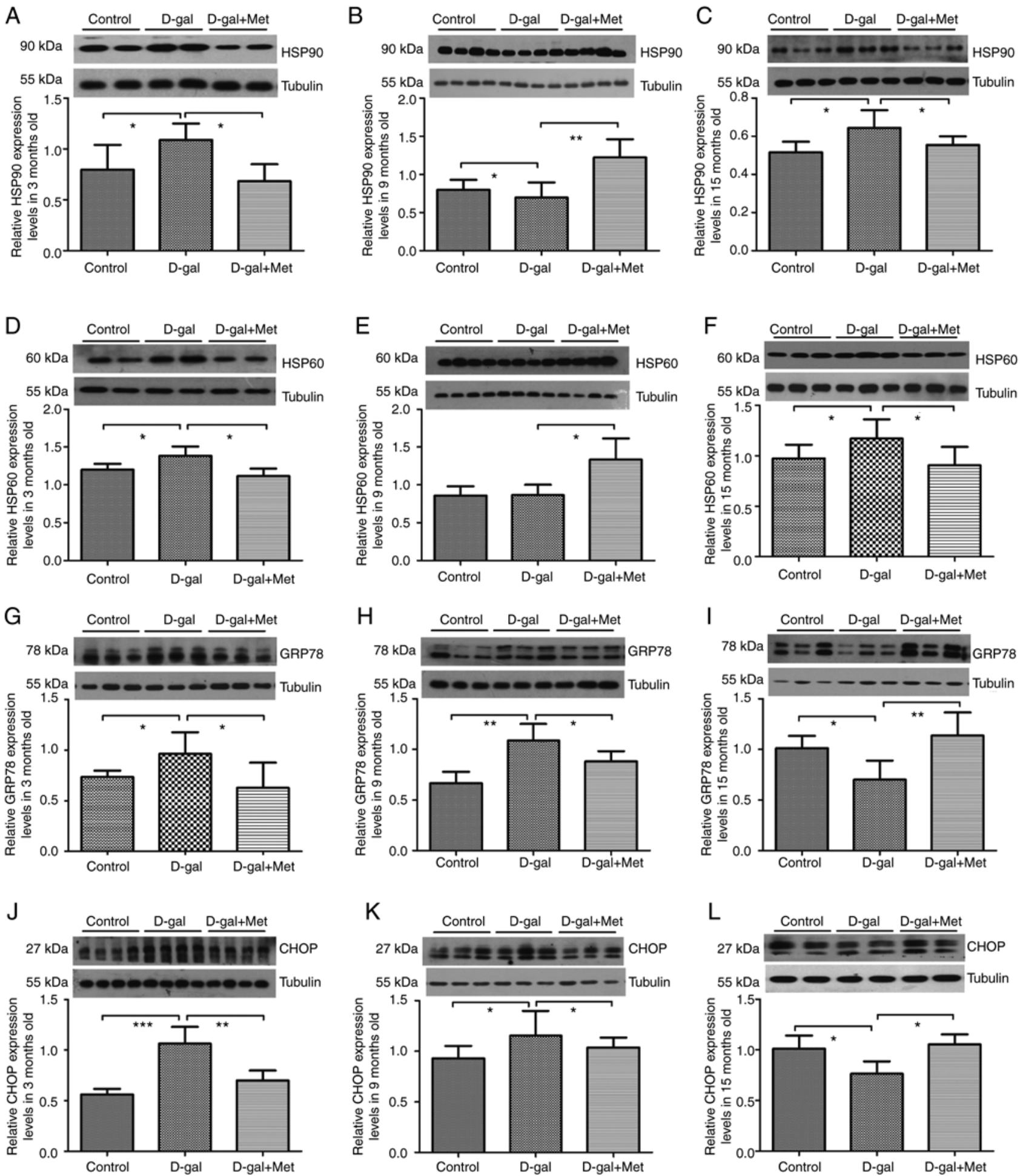

Figure 9. Regulation of ER-UPR and MT-UPR in vitro and in vivo. Western blot analysis of (A-C) HSP90, (D-F) HSP60, (G-I) GRP78 and (J-L) CHOP expression in each group of rats. ${ }^{*} \mathrm{P}<0.05,{ }^{* * *} \mathrm{P}<0.01,{ }^{* * *} \mathrm{P}<0.001$. The data are displayed as the means $\pm \mathrm{SD} ; \mathrm{n}=6$ for each subgroup. $\mathrm{D}$-gal, $\mathrm{D}$-galactose; Met, metformin.

\section{Discussion}

D-gal accelerates the process of age-related neurodegeneration. Mice treated with D-gal exhibited oxidant damage in the blood, brain and liver, and D-gal treatment in mice was confirmed as a successful mimetic aging model (31). Our previous study confirmed that $\mathrm{D}$-gal injection induced a mimetic aging effect in the inner ear of Wistar rats (32).
Eight weeks of D-gal treatment successfully established an ideal mimetic SD rat model of presbycusis in our previous study (33).

In this study, a significant decline in the levels of antioxidants was noted after the injection of D-gal, as observed in several neurodegenerative disorders. ROS production increases with age, and it is known that a lack of proteostasis, oxidative stress and the dysfunction of organelles 
A
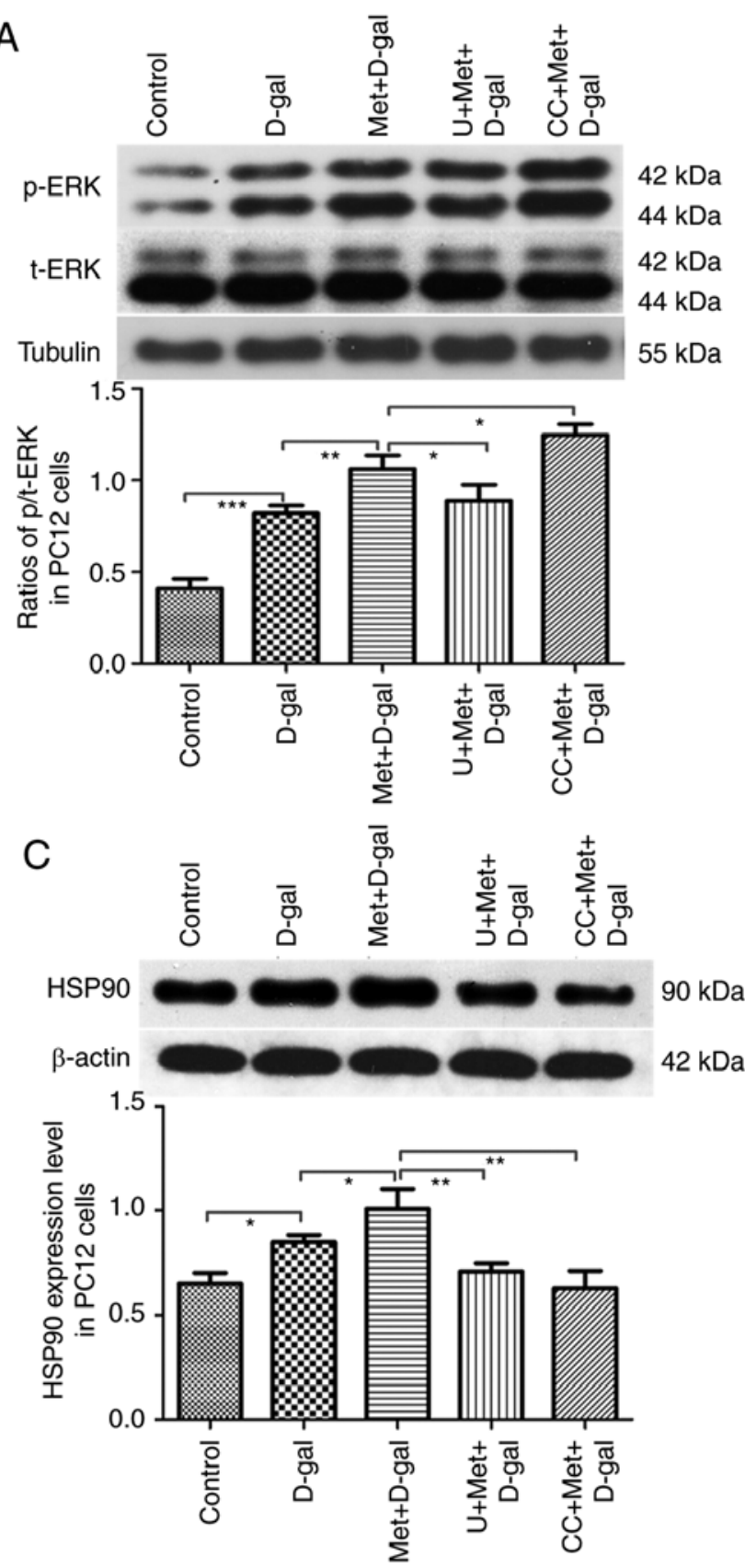
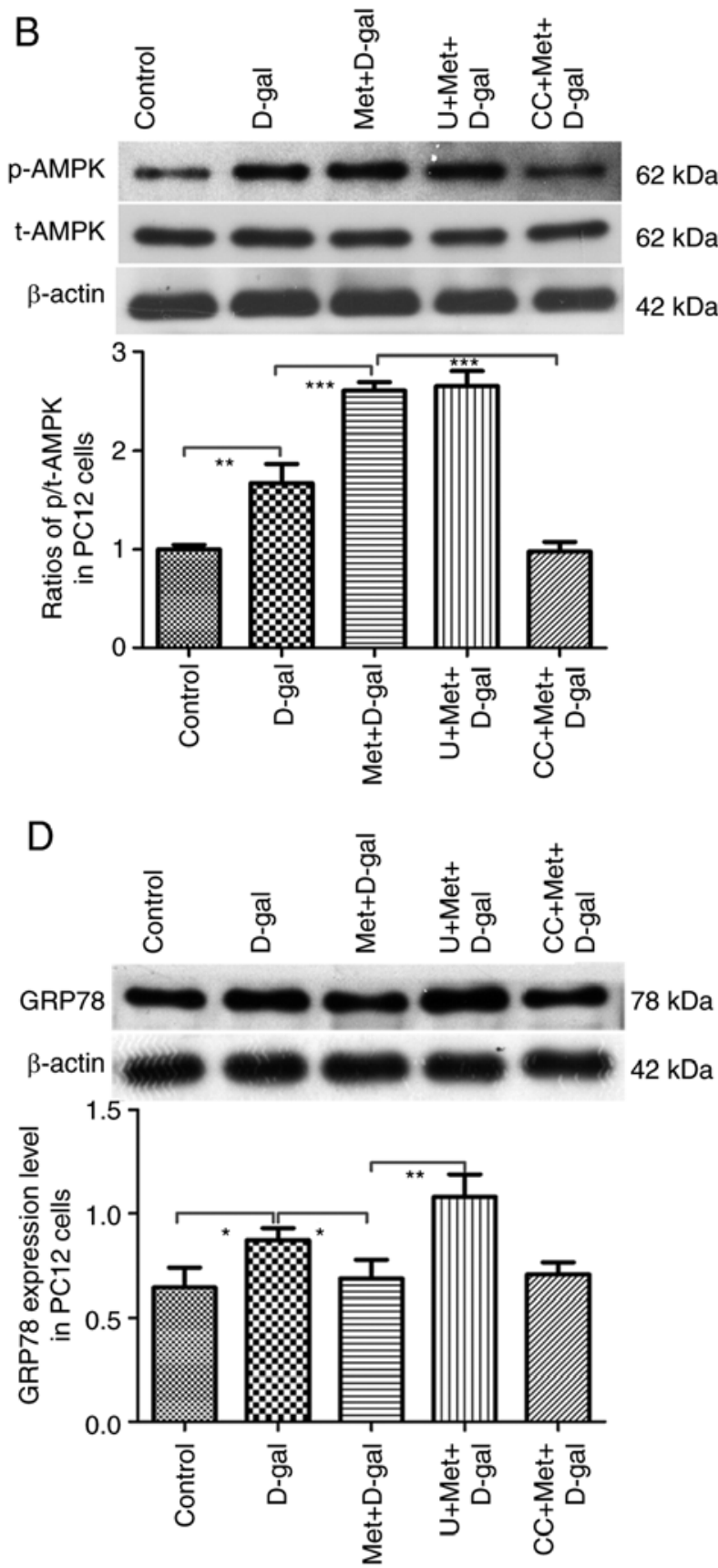

Figure 10. Western blot analysis of the effects of metformin on the (A and B) ERK1/2/AMPK and the (C and D) UPR in PC12 cells. "P<0.05, ** P $<0.01$, ${ }_{* * * *} \mathrm{P}<0.001$. The data are presented as the means $\pm \mathrm{SD} ; \mathrm{n}=6$ for each subgroup. D-gal, D-galactose; Met, metformin; U, U0126; CC, compound C.

are critical factors leading to the onset of age-related diseases (34-36). The mitochondria play a central role in a number of metabolic tasks, such as the regulation of cellular metabolism, cellular proliferation regulation, calcium signaling and hormonal signaling; however, the function of the mitochondria decreases with age (37-39). Studies have suggested that the MT-UPR is triggered when mitochondrial DNA and nuclear DNA are not balanced $(40,41)$. Multiple studies have indicated that there is a strong associatoin between the UPR and longevity, physiology, and age-related diseases $(40,42,43)$. The ROS signal is a major modulator in the process of aging (44); however, the effect of the MT-UPR on longevity does not depend on the ROS signal (40). The 4,834-bp mtDNA deletion in rats is similar to the 3,867-bp deletion in mice and the 4,977-bp deletion in humans; these deletions are also known as CDs, and they have been reported to be the most common age-related mtDNA damage and are used as biomarkers of aging (45-47). Animal models of aging established by our laboratory can trigger oxidative stress and increase the accumulation of the $\mathrm{CD}$ in the peripheral and central auditory system $(19,48)$. In this study, we also found the same results in PC12 cells, and the CD accumulated more readily in aged rats. The mitochondrial ultrastructure was damaged, and flow cytometry data revealed that the decreased mitochondrial membrane permeabilization after the injection of D-gal, which indicated that the number of early apoptotic mitochondria was increased. Both mtDNA mutations and mitochondrial ultrastructural damage in vitro 
A
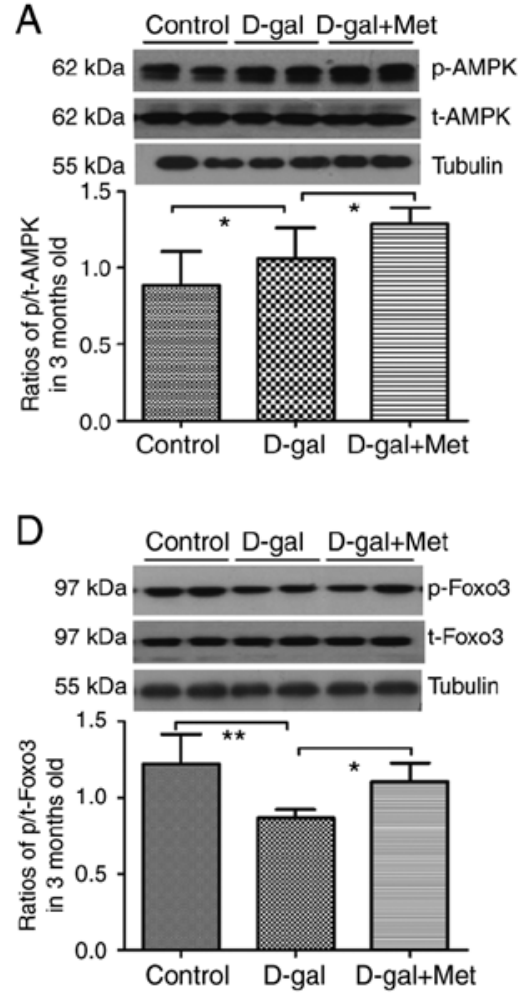

G

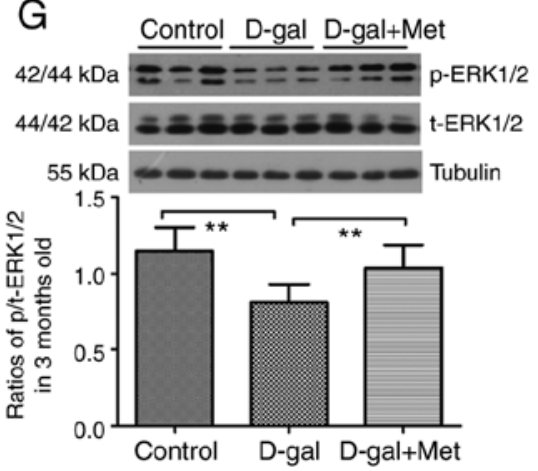

B
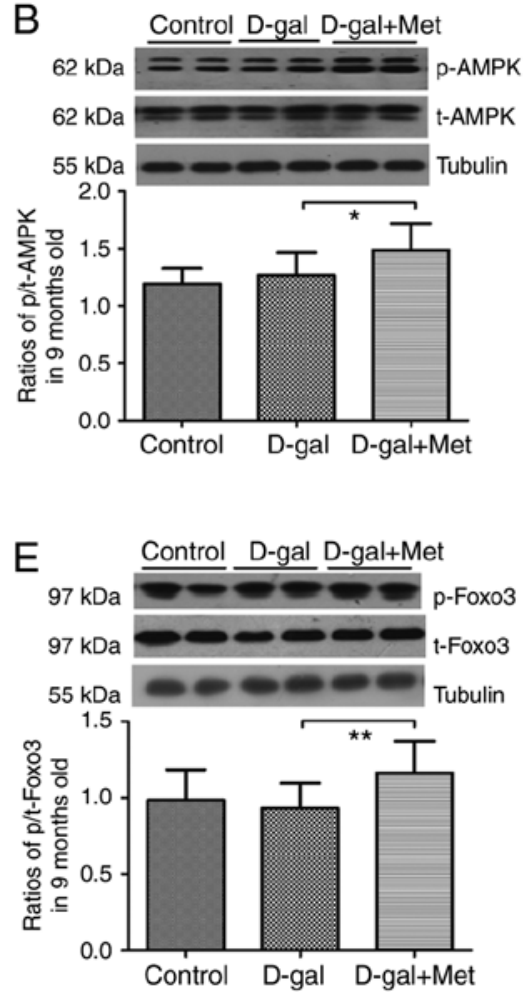

$\mathrm{H}$

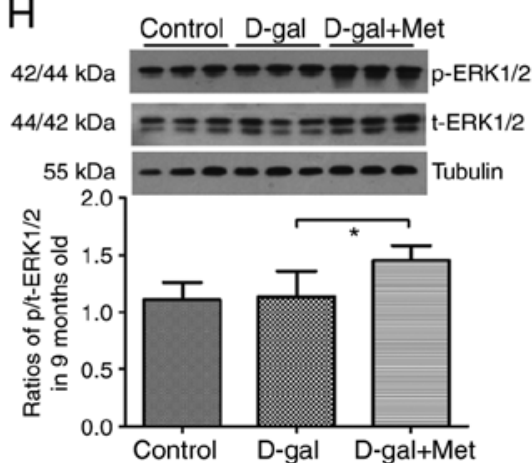

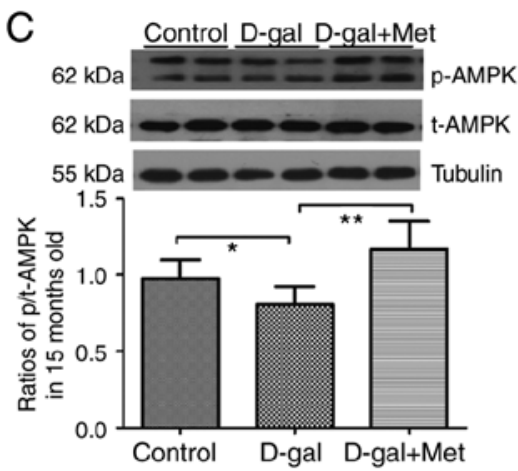

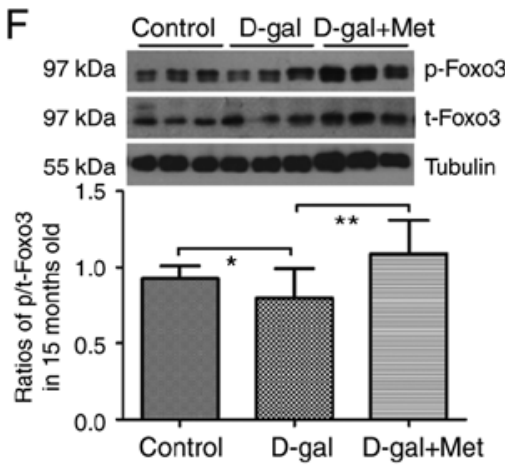

I

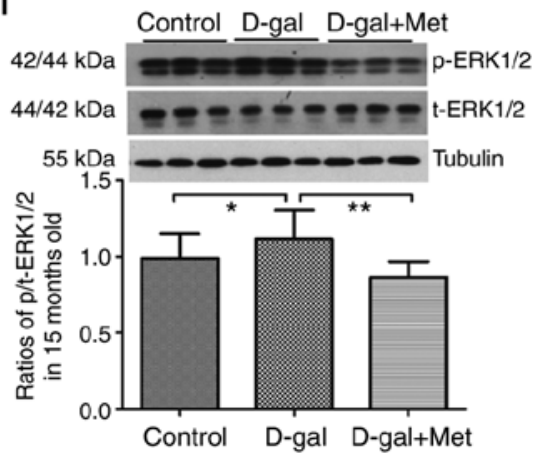

Figure 11. Western blot analysis of the effects of metformin on the AMPK/Foxo3/ERK1/2 signaling pathways [(A-C) AMPK, (D-F) Foxo3 and (G-I) ERK1/2] in aging rats. ${ }^{*} \mathrm{P}<0.05,{ }^{* *} \mathrm{P}<0.01$. The data are presented as the means $\pm \mathrm{SD} ; \mathrm{n}=6$ for each subgroup.

and in vivo weaken mitochondrial function. Herein, significant decreases in the number of neurons and severe apoptosis were observed after the injection of D-gal. At the same time, the p53- and caspase-3-dependent apoptosis pathways were activated by $\mathrm{D}$-gal.

The UPR protects organelle protein homeostasis in the aging brain. Recently, a number of researchers have attempted to clarify the mechanism sof the UPR in maintaining homeostasis (49). Although it has been reported that the UPR is involved in age-related neurodegenerative diseases (27); however, the specific role and function of the UPR in various diseases is fairly complex and controversial.

This study indicated an increase in UPR in the D-gal groups. The accumulation of unfolded or misfolded proteins induced by chronic D-gal injection resulted in the elevation in the levels of the stress response-related proteins, HSP90, HSP60, GRP78 and CHOP. In some cases, the aberrant accumulation of UPR-related proteins can be a dangerous signal to activate the inflammatory response (50) and shorten lifespan (40). In this study, hearing ability, the morphology of organelles and multiple functions were improved by achieving a balance of the UPR by metformin treatment. Notably, in the 15-month-old D-gal group, GRP78 and CHOP expression decreased significantly, and greater apoptosis and significant neuronal death were observed. This finding may illustrate that aged rats cannot deal with chronic and excessive stress from D-gal and that ER cannot trigger a sufficient UPR to remove or degrade the unfolded or misfolded proteins. Our results are in accordance with those of other studies that the proper activation and mild stimulation of the UPR can ameliorate metabolic and age-related diseases, yet the improper or excessive activation of the UPR can release harmful signals (50-52). These results indicate that ER-UPR in aged rats cannot cope with long-term persistent oxidative stress or trigger adequate UPR signals to combat the stress of unfolded proteins. Our data also indicate that the UPR is time-dependent and that metformin suppresses the overexpressed MT-UPR in rats 
following exposure to D-gal, whereas metformin activates the ER-UPR, which is beneficial for healthy brain metabolism, in aged rat brains.

Metformin protects neurons via the UPR through the AMPK/ERK1/2 signaling pathways. In this study, metformin significantly increased antioxidant capability in vitro and in vivo. Other researchers also reported that metformin exerts antioxidant effects on the brain during oxidative stress (53). In this study, a significant decrease in the occurrence of CD was observed in rats treated with metformin and in senescent PC12 cells. Additionally, metformin alleviated the level of apoptosis and changes in ultrastructural morphology through the AMPK and ERK1/2 pathways. Metformin significantly decreased neuronal apoptosis and reversed abnormal neuronal damage; thus, this study indicated that metformin afforded a useful benefit, maintaining a healthy state and delaying the aging process of the auditory system.

Our data not only confirmed that UPR pathways are potential regulating points for the effects of metformin on proteostasis, but also indicated that the inhibition of AMPK or ERK1/2 can influence the UPR. Our investigations revealed long-term changes in AMPK and ERK1/2 signaling in the auditory cortex and in a cellular model of senescence.

ERK1/2 is a member of the MAPK family and integrates external signals into signaling events, promoting a large amount of cell growth and proliferation (54). In many cases, the phosphorylation of ERK1/2 results in the activation of its kinase activity and leads to prosurvival factors $(55,56)$. By contrast, others have reported that ERK1/2 is often related to cell death and apoptosis (57-60). The level of phosphorylated ERK1/2 and its specific function vary with different stimuli, stimulus methods and stimulus duration. In fact, the role of ERK $1 / 2$ in the aging process pf the brain remains unknown. A few studies have indicated that the phosphorylation of ERK1/2 in brain tissue decreases with age $(61,62)$. This study found that oxidative stress or unfolded protein stress triggered by D-gal deactivated ERK1/2 in 3-month-old rats, yet metformin recovered the phosphorylation levels to regulate cell proliferation and differentiation in rat brains. However, in 15-month-old rats, the results revealed the overexpression of $\mathrm{p}-\mathrm{ERK} 1 / 2$ in aged D-gal-treated rats. This finding was consistent with the changes observed in the morphological and functional experiments. The persistent activation of ERK1/2 contributes to oxidative toxicity and induces cell death (63), and metformin markedly reverses phosphorylation to protect neurons from apoptosis or death. In our in vitro experimetns, D-gal and metformin activated ERK1/2 and AMPK phosphorylation. To our surprise, AMPK inhibition slightly increased the phosphorylation level of ERK1/2, indicating that the AMPK pathway can partially inhibit the ERK1/2 pathway in $\mathrm{PC} 12$ cells.

The crosstalk between the AKT signaling pathway and the ERK1/2 signaling pathway is dynamic. It has been reported that ERK1/2 activation is accompanied by a gradual decrease in both AKT levels and apoptosis (64), and phosphorylated AKT inhibits the activation of ERK1/2 (65). The findings of this study that increased levels of AMPK in rat brains were accompanied by a decrease in ERK1/2 and that an increase in ERK1/2 was accompanied by a decrease in AMPK. However, whether metformin suppresses ERK1/2 activity in aged rats through the regulation of AMPK or through UPR signaling requires further investigation. Our findings also indicated that Foxo $3 \alpha$ may be a regulator of the protection of neuronal survival against $\mathrm{D}$-gal by metformin.

In conclusion, the present data suggest that D-gal injection induces multiple molecular and functional changes, oxidative stress, mitochondrial dysfunction and abnormal apoptosis in the brain similar to natural aging. Metformin regulates the UPR to promote neuronal survival and cellular homeostasis, ultimately resulting in the enhanced hearing ability of D-gal-treated rats. The protective effect of metformin involves the regulation of the UPR through the AMPK/ERK1/2 pathways. Our studies demonstrated a crosstalk between the AMPK/ERK1/2 and UPR signaling pathways. Metformin-induced cell protection in vitro and in vivo may provide novel therapeutic targets for postponing the aging process of the brain and suggest that metformin may be a potential therapeutic treatment for age-related diseases.

\section{Acknowledgements}

Not applicable.

\section{Funding}

This study was supported by research grants from the National Natural Science Foundations of China (nos. 81873700 and 81230021).

\section{Availability of data and materials}

The analyzed datasets generated during the present study are available from the corresponding author on reasonable request.

\section{Authors' contributions}

$\mathrm{HC}, \mathrm{WK}$ and WJK conceived and designed the experiments; $\mathrm{HC}, \mathrm{BH}, \mathrm{YH}, \mathrm{XZ}, \mathrm{ZH}, \mathrm{XC}, \mathrm{HS}, \mathrm{JY}, \mathrm{YL}$ and XY performed the experiments; $\mathrm{HC}, \mathrm{WK}$ and WJK analyzed the data; $\mathrm{HC}, \mathrm{BH}$, YH, WK and WJK wrote the manuscript. All the authors have read and approved the final version of this manuscript.

\section{Ethics approval and consent to participate}

All experimental procedures involving the care of animals were performed in strict accordance with the recommendations of the Guide for the Care and Use of Laboratory Animals of the National Institutes of Health (14). The protocol was under the supervision of the Committee on the Ethics of Animal Experiments of Huazhong University of Science and Technology (Wuhan, China; permit no. IACUC S2219).

\section{Patient consent for publication}

Not applicable.

\section{Competing interests}

The authors declare that they have no competing interests. 


\section{References}

1. Du Z, Yang Y, Hu Y, Sun Y, Zhang S, Peng W, Zhong Y, Huang X and Kong W: A long-term high-fat diet increases oxidative stress, mitochondrial damage and apoptosis in the inner ear of D-galactose-induced aging rats. Hear Res 287: 15-24, 2012.

2. Gates GA and Mills JH: Presbycusis. Lancet 366: 1111-1120, 2005.

3. Humes LE, Dubno JR, Gordon-Salant S, Lister JJ, Cacace AT, Cruickshanks KJ, Gates GA, Wilson RH and Wingfield A: Central presbycusis: A review and evaluation of the evidence. J Am Acad Audiol 23: 635-666, 2012.

4. Taylor RC and Dillin A: Aging as an event of proteostasis collapse. Cold Spring Harb Perspect Biol 3: a004440, 2011.

5. Jensen MB and Jasper H: Mitochondrial proteostasis in the control of aging and longevity. Cell Metab 20: 214-225, 2014.

6. Li J, Akil O, Rouse SL, McLaughlin CW, Matthews IR Lustig LR, Chan DK and Sherr EH: Deletion of Tmtc4 activates the unfolded protein response and causes postnatal hearing loss. J Clin Invest 128: 5150-5162, 2018.

7. Chaube R: Can UPR integrate fasting and stem cell regeneration? FRONT CHEM 3: 5, 2015.

8. Duran-Aniotz C, Martínez G and Hetz C: Memory loss in Alzheimer's disease: Are the alterations in the UPR network involved in the cognitive impairment? Front Aging Neurosci 6 : $8,2014$.

9. Woehlbier U and Hetz C: Modulating stress responses by the UPRosome: A matter of life and death. Trends Biochem Sci 36 : 329-337, 2011

10. Zhao RR, Xu XC, Xu F, Zhang WL, Zhang WL, Liu LM and Wang WP: Metformin protects against seizures, learning and memory impairments and oxidative damage induced by pentylenetetrazole-induced kindling in mice. Biochem Biophys Res Commun 448: 414-417, 2014.

11. Patil SP, Jain PD, Ghumatkar PJ, Tambe R and Sathaye S: Neuroprotective effect of metformin in MPTP-induced Parkinson's disease in mice. Neuroscience 277: 747-754, 2014.

12. Liang D, Song Z, Liang W, Li Y and Liu S: Metformin inhibits TGF-beta 1-induced MCP-1 expression through BAMBI-mediated suppression of MEK/ERK1/2 signalling. Nephrology (Carlton) 24: 481-488, 2019.

13. Tao L, Li D, Liu H, Jiang F, Xu Y, Cao Y, Gao R and Chen G: Neuroprotective effects of metformin on traumatic brain injury in rats associated with NF- $\mathrm{BB}$ and MAPK signaling pathway. Brain Res Bull 140: 154-161, 2018.

14. National Research Council (US) Committee for the Update of the Guide for the Care and Use of Laboratory Animals: Guide for the Care and Use of Laboratory Animals. National Academies Press, Washington, DC, 2011.

15. Khallaghi B, Safarian F, Nasoohi S, Ahmadiani A and Dargahi L: Metformin-induced protection against oxidative stress is associated with AKT/mTOR restoration in PC12 cells. Life Sci 148 286-292, 2016

16. Jiang HY, Yang Y, Zhang YY, Xie Z, Zhao XY, Sun Y and Kong WJ: The dual role of poly(ADP-ribose) polymerase-1 in modulating parthanatos and autophagy under oxidative stress in rat cochlear marginal cells of the stria vascularis. Redox Biol 14 $361-370,2018$

17. Itahana K, Campisi J and Dimri GP: Methods to detect biomarkers of cellular senescence: The senescence-associated beta-galactosidase assay. Methods Mol Biol 371: 21-31, 2007.

18. Wu X, Wang Y, Sun Y, Chen S, Zhang S, Shen L, Huang X, Lin X and Kong W: Reduced expression of Connexin26 and its DNA promoter hypermethylation in the inner ear of mimetic aging rats induced by d-galactose. Biochem Biophys Res Commun 452: 340-346, 2014

19. Kong WJ, Hu YJ, Wang Q, Wang Y, Han YC, Cheng HM, Kong W and Guan MX: The effect of the mtDNA4834 deletion on hearing. Biochem Biophys Res Commun 344: 425-430, 2006.

20. Nicklas JA, Brooks EM, Hunter TC, Single R and Branda RF: Development of a quantitative PCR (TaqMan) assay for relative mitochondrial DNA copy number and the common mitochondrial DNA deletion in the rat. Environ Mol Mutagen 44 313-320, 2004

21. Livak KJ and Schmittgen TD: Analysis of relative gene expression data using real-time quantitative PCR and the 2(-Delta Delta C(T)) method. Methods 25: 402-408, 2001.
22. Sun HY, Hu YJ, Zhao XY, Zhong Y, Zeng LL, Chen XB, Yuan J, Wu J, Sun Y, Kong W and Kong WJ: Age-related changes in mitochondrial antioxidant enzyme Trx2 and TXNIP-Trx2-ASK1 signal pathways in the auditory cortex of a mimetic aging rat model: Changes to $\operatorname{Tr} x 2$ in the auditory cortex. FEBS J 282 : 2758-2774, 2015

23. Xia MY, Zhao XY, Huang QL, Sun HY, Sun C, Yuan J, He C, Sun Y, Huang X, Kong W and Kong WJ: Activation of Wnt $/ \beta$-catenin signaling by lithium chloride attenuates d-galactose-induced neurodegeneration in the auditory cortex of a rat model of aging. FEBS Open Bio 7: 759-776, 2017.

24. Tian YY, Jiang B, An LJ and Bao YM: Neuroprotective effect of catalpol against MPP(+)-induced oxidative stress in mesencephalic neurons. Eur J Pharmacol 568: 142-148, 2007.

25. Kong WJ, Wang Y, Wang Q, Hu YJ, Han YC and Liu J: The relation between D-galactose injection and mitochondrial DNA 4834 bp deletion mutation. Exp Gerontol 41: 628-634, 2006.

26. Zeng L, Yang Y, Hu Y, Sun Y, Du Z, Xie Z, Zhou T and Kong W: Age-related decrease in the mitochondrial sirtuin deacetylase Sirt 3 expression associated with ROS accumulation in the auditory cortex of the mimetic aging rat model. PLoS One 9: e88019, 2014

27. Bernales S, Soto MM and McCullagh E: Unfolded protein stress in the endoplasmic reticulum and mitochondria: A role in neurodegeneration. Front Aging Neurosci 4: 5, 2012.

28. Wang W, Sun Y, Chen S, Zhou X, Wu X, Kong W and Kong W: Impaired unfolded protein response in the degeneration of cochlea cells in a mouse model of age-related hearing loss. Exp Gerontol 70: 61-70, 2015.

29. Chen J, Huang Z, Wu X, Kang J, Ren Y, Gao W, Lu X, Wang J, Ding W, Nakabeppu Y, et al: Oxidative stress induces different tissue dependent effects on Mutyh-deficient mice. Free Radic Biol Med 143: 482-493, 2019.

30. Mendoza MC,Er EE and Blenis J: The Ras-ERK and PI3K-mTOR pathways: Cross-talk and compensation. Trends Biochem Sci 36: 320-328, 2011.

31. Ho SC, Liu JH and Wu RY: Establishment of the mimetic aging effect in mice caused by D-galactose. Biogerontology 4: 15-18, 2003 .

32. Kong W, Hu Y, Wang Q, Xu L, Wang Y, Han Y, Li J, Liu B and Kong W: Establishment of model with inner ear mimetic aging and mtDNA 4834 bp deletion in rats. Lin Chuang Er Bi Yan Hou Ke Za Zhi 20: 888-890, 893, 2006 (In Chinese).

33. Zhong Y, Hu Y, Peng W, Sun Y, Yang Y, Zhao X, Huang X, Zhang $\mathrm{H}$ and Kong W: Age-related decline of the cytochrome $\mathrm{c}$ oxidase subunit expression in the auditory cortex of the mimetic aging rat model associated with the common deletion. Hear Res 294: 40-48, 2012.

34. Balaban RS, Nemoto S and Finkel T: Mitochondria, oxidants, and aging. Cell 120: 483-495, 2005.

35. Lin MT and Beal MF: Mitochondrial dysfunction and oxidative stress in neurodegenerative diseases. Nature 443: 787-795, 2006.

36. Sands WA, Page MM and Selman C: Proteostasis and ageing: Insights from long-lived mutant mice. J Physiol 595: 6383-6390, 2017.

37. McBride HM, Neuspiel M and Wasiak S: Mitochondria: More than just a powerhouse. Curr Biol 16: R551-R560, 2006.

38. Klinge CM: Estrogenic control of mitochondrial function and biogenesis. J Cell Biochem 105: 1342-1351, 2008.

39. Weinberg F and Chandel NS: Mitochondrial metabolism and cancer. Ann N Y Acad Sci 1177: 66-73, 2009.

40. Houtkooper RH, Mouchiroud L, Ryu D, Moullan N, Katsyuba E, Knott G, Williams RW and Auwerx J: Mitonuclear protein imbalance as a conserved longevity mechanism. Nature 497: 451-457, 2013.

41. Yoneda T, Benedetti C, Urano F, Clark SG, Harding HP and Ron D: Compartment-specific perturbation of protein handling activates genes encoding mitochondrial chaperones. J Cell Sci 117: 4055-4066, 2004

42. Shpilka T and Haynes CM: The mitochondrial UPR: Mechanisms, physiological functions and implications in ageing. Nat Rev Mol Cell Biol 19: 109-120, 2018.

43. Beck JS, Mufson EJ and Counts SE: Evidence for mitochondrial UPR gene activation in familial and sporadic Alzheimer's disease. Curr Alzheimer Res 13: 610-614, 2016.

44. Zarse K, Schmeisser S, Groth M, Priebe S, Beuster G, Kuhlow D, Guthke R, Platzer M, Kahn CR and Ristow M: Impaired insulin/IGF1 signaling extends life span by promoting mitochondrial L-proline catabolism to induce a transient ROS signal. Cell Metab 15: 451-465, 2012. 
45. Mohamed SA, Hanke T, Erasmi AW, Bechtel MJ, Scharfschwerdt M, Meissner C, Sievers HH and Gosslau A: Mitochondrial DNA deletions and the aging heart. Exp Gerontol 41: 508-517, 2006

46. Chen B, Zhong Y, Peng W, Sun Y, Hu YJ, Yang Y and Kong WJ: Increased mitochondrial DNA damage and decreased base excision repair in the auditory cortex of D-galactose-induced aging rats. Mol Biol Rep 38: 3635-3642, 2011.

47. Zeng Z, Zhang Z, Yu H, Corbley MJ, Tang Z and Tong T: Mitochondrial DNA deletions are associated with ischemia and aging in Balb/c mouse brain. J Cell Biochem 73: 545-553, 1999.

48. Chen B, Zhong Y, Peng W, Sun Y and Kong WJ: Age-related changes in the central auditory system: Comparison of D-galactose-induced aging rats and naturally aging rats. Brain Res 1344: 43-53, 2010.

49. Eletto D, Chevet E, Argon Y and Appenzeller-Herzog C: Redox controls UPR to control redox. J Cell Sci 127: 3649-3658, 2014.

50. Hill S and Van Remmen H: Mitochondrial stress signaling in longevity: A new role for mitochondrial function in aging. Redox Biol 2: 936-944, 2014.

51. Cohen E, Bieschke J, Perciavalle RM, Kelly JW and Dillin A: Opposing activities protect against age-onset proteotoxicity. Science 313: 1604-1610, 2006.

52. Labunskyy VM, Gerashchenko MV, Delaney JR, Kaya A, Kennedy BK, Kaeberlein M and Gladyshev VN: Lifespan extension conferred by endoplasmic reticulum secretory pathway deficiency requires induction of the unfolded protein response. PLoS Genet 10: e1004019, 2014

53. Correia S, Carvalho C, Santos MS, Proença T, Nunes E, Duarte AI, Monteiro P, Seiça R, Oliveira CR and Moreira PI: Metformin protects the brain against the oxidative imbalance promoted by type 2 diabetes. Med Chem 4: 358-364, 2008.

54. Kurioka T, Matsunobu T, Satoh Y, Niwa K, Endo S, Fujioka M and Shiotani A: ERK2 mediates inner hair cell survival and decreases susceptibility to noise-induced hearing loss. Sci Rep 5: $16839,2015$.

55. Arthur DB, Georgi S, Akassoglou K and Insel PA: Inhibition of apoptosis by P2Y2 receptor activation: Novel pathways for neuronal survival. J Neurosci 26: 3798-3804, 2006.

56. Xia Z, Dickens M, Raingeaud J, Davis RJ and Greenberg ME: Opposing effects of ERK and JNK-p38 MAP kinases on apoptosis. Science 270: 1326-1331, 1995.
57. Stanciu M, Wang Y, Kentor R, Burke N, Watkins S, Kress G, Reynolds I, Klann E, Angiolieri MR, Johnson JW and DeFranco DB: Persistent activation of ERK contributes to glutamate-induced oxidative toxicity in a neuronal cell line and primary cortical neuron cultures. J Biol Chem 275: 12200-12206, 2000.

58. Liu L, Cao Y, Chen C, Zhang X, McNabola A, Wilkie D, Wilhelm S, Lynch $\mathrm{M}$ and Carter C: Sorafenib blocks the $\mathrm{RAF} / \mathrm{MEK} / \mathrm{ERK}$ pathway, inhibits tumor angiogenesis, and induces tumor cell apoptosis in hepatocellular carcinoma model PLC/PRF/5. Cancer Res 66: 11851-11858, 2006.

59. Tavares R and Pathak SK: Helicobacter pylori Secreted protein HP1286 triggers apoptosis in macrophages via TNF-independent and ERK MAPK-dependent pathways. Front Cell Infect Microbiol 7: 58, 2017.

60. Feng X, Sun T, Bei Y, Ding S, Zheng W, Lu Y and Shen P: S-nitrosylation of ERK inhibits ERK phosphorylation and induces apoptosis. Sci Rep 3: 1814, 2013.

61. Allen EN, Potdar S, Tapias V, Parmar M, Mizuno CS, Rimando A and Cavanaugh JE: Resveratrol and pinostilbene confer neuroprotection against aging-related deficits through an ERK1/2-dependent mechanism. J Nutr Biochem 54: 77-86, 2018.

62. Zhen X, Uryu K, Cai G, Johnson GP and Friedman E: Age-associated impairment in brain MAPK signal pathways and the effect of caloric restriction in Fischer 344 rats. J Gerontol A Biol Sci Med Sci 54: B539-B548, 1999.

63. Zhuang S and Schnellmann RG: A death-promoting role for extracellular signal-regulated kinase. J Pharmacol Exp Ther 319: 991-997, 2006.

64. Sinha D, Bannergee S, Schwartz JH, Lieberthal W and Levine JS: Inhibition of ligand-independent ERK1/2 activity in kidney proximal tubular cells deprived of soluble survival factors up-regulates Akt and prevents apoptosis. J Biol Chem 279: 10962-10972, 2004.

65. Moelling K, Schad K, Bosse M,Zimmermann S and Schweneker M: Regulation of Raf-Akt cross-talk. J Biol Chem 277: 31099-31106, 2002.

This work is licensed under a Creative Commons Attribution-NonCommercial-NoDerivatives 4.0 International (CC BY-NC-ND 4.0) License. 